\title{
A CHARACTERIZATION OF RIGHT COIDEALS OF QUOTIENT TYPE AND ITS APPLICATION TO CLASSIFICATION OF POISSON BOUNDARIES
}

\author{
REIJI TOMATSU
}

\begin{abstract}
Let $\mathbb{G}$ be a co-amenable compact quantum group. We show that a right coideal of $\mathbb{G}$ is of quotient type if and only if it is the range of a conditional expectation preserving the Haar state and is globally invariant under the left action of the dual discrete quantum group. We apply this result to theory of Poisson boundaries introduced by Izumi for discrete quantum groups and generalize a work of Izumi-Neshveyev-Tuset on $S U_{q}(N)$ for coamenable compact quantum groups with the commutative fusion rules. More precisely, we prove that the Poisson integral is an isomorphism between the Poisson boundary and the right coideal of quotient type by maximal quantum subgroup of Kac type. In particular, the Poisson boundary and the quantum flag manifold are isomorphic for any $q$-deformed classical compact Lie group.
\end{abstract}

\section{INTRODUCTION}

Since Woronowicz introduced the axiomatic compact quantum groups [30], they have attracted a growing interest of many researchers as a framework to describe new types of symmetries. In this paper, we study two typical examples of ergodic actions of compact quantum groups, namely right coideals and Poisson boundaries.

Let $\mathbb{G}$ be a compact quantum group. A right coideal is a von Neumann subalgebra of the function algebra on $\mathbb{G}$ which is globally invariant under the right translation action of $\mathbb{G}$. Taking the fixed point algebra of a left action of a quantum subgroup gives an example of a right coideal. We say that such a right coideal is of quotient type. When $\mathbb{G}$ is an ordinary group, it is well-known that all the right coideals are of quotient type. However, when $\mathbb{G}$ is a quantum group, not all the right coideals are realized as quotients [18], [19], 23]. This fact presents a contrast between the quantum groups and the ordinary ones.

Another difference occurs in behaviors of infinite tensor product actions. In the ordinary case, such an action is minimal and, in particular, the relative commutant of the fixed point algebra is trivial. However, this is not the case for quantum groups. In [10], Izumi has described this contrast by introducing the notion of a Poisson boundary of a dual discrete quantum group. More precisely, he has shown that the Poisson boundary is isomorphic to the relative commutant of the fixed point algebra. Moreover he has also studied the Poisson boundary of the dual of $S U_{q}(2)$ and shown a striking result that the Poisson boundary is

2000 Mathematics Subject Classification. Primary 46L65; Secondary 46L55. 
isomorphic to the standard Podleś sphere $L^{\infty}\left(\mathbb{T} \backslash S U_{q}(2)\right)$ introduced in [18], [19]. On one hand, this result has led to a conjecture that the Poisson boundary is isomorphic to the quantum flag manifold for any $q$-deformed classical compact Lie group. For $S U_{q}(N)$, the conjecture was confirmed affirmatively in [12]. Note that a $q$-deformed classical compact Lie group is co-amenable, that is, the dual discrete quantum group is amenable in the sense of [3]. On the other hand for non-amenable cases, the Poisson and Martin boundaries [17] of universal orthogonal discrete quantum groups are studied in [25] and [26].

In this paper, we first characterize when a right coideal is of quotient type and second apply the characterization to determine Poisson boundaries of amenable discrete quantum groups with the commutative fusion rules. A right coideal of quotient type has the following two properties. The first one is the expectation property, namely, existence of a normal conditional expectation preserving the Haar state from the function algebra. The second one is the coaction symmetry which means that the left action of the dual preserves the right coideal. Assuming amenability of the dual, we can prove the following theorem (Theorem 3.18).

Theorem 1. Let $\mathbb{G}$ be a co-amenable compact quantum group and $B \subset L^{\infty}(\mathbb{G})$ a right coideal. Then $B$ is of quotient type if and only if $B$ has the expectation property and the coaction symmetry.

Next we study a Poisson boundary of an amenable discrete quantum group. In order to compute a Poisson boundary, we present an approach which differs from that of [12. The key point of our proof is to construct an "inverse" of the Poisson integral. Although this strategy is the same as the one taken in [12], we do it by utilizing not the Berezin transforms but an invariant mean of a dual discrete quantum group. Then using Theorem 1, we show that the Poisson boundary is isomorphic to a right coideal of quotient type by a quantum subgroup. Moreover, we can specify the quantum subgroup which is the maximal quantum subgroup of Kac type with respect to inclusions. After this work was done, we learned from $\mathrm{S}$. Vaes that the notion has been already introduced as the canonical Kac quotient in [20]. Our main result is the following theorem (Theorem 4.8).

Theorem 2. Let $\mathbb{G}$ be a co-amenable compact quantum group. Assume that its fusion algebra is commutative. Then the following statements hold.

(1) There exists a unique maximal quantum subgroup of Kac type $\mathbb{H}$.

(2) The Poisson integral $\Theta: L^{\infty}(\mathbb{H} \backslash \mathbb{G}) \rightarrow H^{\infty}(\widehat{\mathbb{G}})$ is an isomorphism.

In particular, $\mathbb{G}$ is of Kac type if and only if $H^{\infty}(\widehat{\mathbb{G}})=\mathbb{C}$. This yields the minimality of an infinite tensor product actions of $\mathbb{G}$. For a $q$-deformed classical compact Lie group $\mathbb{G}_{q}$, the maximal quantum subgroup of Kac type is the maximal torus $\mathbb{T}$. Therefore, we obtain the following result (Corollary 4.11).

Theorem 3. Let $\mathbb{G}_{q}$ be the $q$-deformation of a classical compact Lie group $\mathbb{G}$. Then the Poisson integral $\Theta: L^{\infty}\left(\mathbb{T} \backslash \mathbb{G}_{q}\right) \rightarrow H^{\infty}\left(\widehat{\mathbb{G}_{q}}\right)$ is an isomorphism. 
Notations. Let $M$ be a von Neumann algebra with predual $M_{*}$. We denote by $M_{*}^{+}$the set of positive functionals in $M_{*}$. For a linear functional $\theta$ on $M$, we define a linear functional $\bar{\theta}$ by $\bar{\theta}(x)=\overline{\theta\left(x^{*}\right)}$ for $x \in M$. For a weight $\phi$ on $M$, we set $n_{\phi}=\left\{x \in M \mid \phi\left(x^{*} x\right)<\infty\right\}, m_{\phi}=n_{\phi}^{*} n_{\phi}$. We denote by $m_{\phi}^{+}$the set of positive elements in $m_{\phi}$. For a linear subspace $X \subset M$, we denote by $\bar{X}^{\mathrm{w}}$ the weak closure of $X$. We denote by $\otimes$ the minimal tensor product for $C^{*}$-algebras and the spatial tensor product for von Neumann algebras.

\section{Preliminaries}

We collect necessary results on compact (discrete) quantum groups.

\subsection{Compact quantum groups.}

Our standard references are [1], [10, [30]. For the notion of a compact quantum group, we adopt the definition in [30, Definition 2.1] as follows:

Definition 2.1. A compact quantum group $\mathbb{G}$ is a pair $\left(C(\mathbb{G}), \delta_{\mathbb{G}}\right)$ which satisfies the following conditions:

(1) $C(\mathbb{G})$ is a separable unital $C^{*}$-algebra.

(2) The map $\delta_{\mathbb{G}}: C(\mathbb{G}) \rightarrow C(\mathbb{G}) \otimes C(\mathbb{G})$ is a coproduct, i.e. it is a faithful unital $*$-homomorphism satisfying the coassociativity condition,

$$
\left(\delta_{\mathbb{G}} \otimes \mathrm{id}\right) \circ \delta_{\mathbb{G}}=\left(\mathrm{id} \otimes \delta_{\mathbb{G}}\right) \circ \delta_{\mathbb{G}} .
$$

(3) The vector spaces $\delta_{\mathbb{G}}(C(\mathbb{G}))(\mathbb{C} \otimes C(\mathbb{G}))$ and $\delta_{\mathbb{G}}(C(\mathbb{G}))(C(\mathbb{G}) \otimes \mathbb{C})$ are dense in $C(\mathbb{G}) \otimes C(\mathbb{G})$.

Let $h_{\mathbb{G}}$ be the Haar state on $C(\mathbb{G})$ which satisfies the invariance condition,

$$
\left(\mathrm{id} \otimes h_{\mathbb{G}}\right)\left(\delta_{\mathbb{G}}(a)\right)=h_{\mathbb{G}}(a) 1=\left(h_{\mathbb{G}} \otimes \mathrm{id}\right)\left(\delta_{\mathbb{G}}(a)\right) \quad \text { for all } a \in C(\mathbb{G}) .
$$

In this paper, we always assume that the Haar states are faithful. If the Haar state is tracial, we say that the compact quantum group is of Kac type [7]. Let $\left(\pi_{h}, L^{2}(\mathbb{G}), \hat{1}_{h}\right)$ be the GNS triple of $h_{\mathbb{G}}$, which consists of the representation, the Hilbert space and the GNS cyclic vector, respectively. We always omit $\pi_{h}$ and regard $C(\mathbb{G})$ as a $C^{*}$-subalgebra of $B\left(L^{2}(\mathbb{G})\right)$. We set a von Neumann algebra $L^{\infty}(\mathbb{G})=\overline{C(\mathbb{G})}$.

The multiplicative unitaries $V_{\mathbb{G}}$ and $W_{\mathbb{G}}$ are defined by

$$
\begin{array}{ll}
W_{\mathbb{G}}^{*}\left(x \hat{1}_{h} \otimes y \hat{1}_{h}\right)=\delta_{\mathbb{G}}(y)\left(x \hat{1}_{h} \otimes \hat{1}_{h}\right) & \text { for } x, y \in C(\mathbb{G}), \\
V_{\mathbb{G}}\left(x \hat{1}_{h} \otimes y \hat{1}_{h}\right)=\delta_{\mathbb{G}}(x)\left(\hat{1}_{h} \otimes y \hat{1}_{h}\right) & \text { for } x, y \in C(\mathbb{G}) .
\end{array}
$$

Then we have the pentagon equalities,

$$
\begin{gathered}
\left(W_{\mathbb{G}}\right)_{12}\left(W_{\mathbb{G}}\right)_{13}\left(W_{\mathbb{G}}\right)_{23}=\left(W_{\mathbb{G}}\right)_{23}\left(W_{\mathbb{G}}\right)_{12}, \\
\left(V_{\mathbb{G}}\right)_{12}\left(V_{\mathbb{G}}\right)_{13}\left(V_{\mathbb{G}}\right)_{23}=\left(V_{\mathbb{G}}\right)_{23}\left(V_{\mathbb{G}}\right)_{12} .
\end{gathered}
$$

Using them, we can extend the coproduct $\delta_{\mathbb{G}}$ to $L^{\infty}(\mathbb{G})$ by

$$
\delta_{\mathbb{G}}(x)=V_{\mathbb{G}}(x \otimes 1) V_{\mathbb{G}}^{*}=\underset{3}{W_{\mathbb{G}}^{*}}(1 \otimes x) W_{\mathbb{G}} \quad \text { for } x \in L^{\infty}(\mathbb{G}) .
$$


The normal state $h_{\mathbb{G}}(x)=\left(x \hat{1}_{h}, \hat{1}_{h}\right), x \in L^{\infty}(\mathbb{G})$ is invariant under $\delta_{\mathbb{G}}$. Since $h_{\mathbb{G}}$ on $C(\mathbb{G})$ has the modular automorphism group [30, Theorem 2.4], $h_{\mathbb{G}}$ is also faithful on $L^{\infty}(\mathbb{G})$. Then the pair $\left(L^{\infty}(\mathbb{G}), \delta_{\mathbb{G}}\right)$ is a von Neumann algebraic compact quantum group in the sense of [16].

Let $H$ be a Hilbert space and $v \in B(H) \otimes L^{\infty}(\mathbb{G})$ a unitary. If $\left(\mathrm{id} \otimes \delta_{\mathbb{G}}\right)(v)=$ $v_{12} v_{13}$, we say that $v$ is a right unitary representation of $\mathbb{G}$. A left unitary representation is similarly defined. For example, the unitaries $W_{\mathbb{G}}$ and $V_{\mathbb{G}}$ are left and right unitary representations, respectively. Let $v \in B(H) \otimes L^{\infty}(\mathbb{G})$ be a unitary representation. Suppose that $T \in B(H)$ satisfies $(T \otimes 1) v=v(T \otimes 1)$. If any such element $T$ must be a scalar, $v$ is said to be irreducible. Any unitary representation is completely decomposable, that is, it is a direct sum of irreducible ones. The set of the equivalence classes of all the irreducible representations is denoted by $\operatorname{Irr}(\mathbb{G})$. For $\pi \in \operatorname{Irr}(\mathbb{G})$, we choose a representation Hilbert space $H_{\pi}$ and an irreducible representation $v_{\pi}=\left(v_{\pi_{i, j}}\right)_{i, j \in I_{\pi}} \in B\left(H_{\pi}\right) \otimes C(\mathbb{G})$. We call an irreducible representation $1 \in \mathbb{C} \otimes L^{\infty}(\mathbb{G})$ the trivial representation and denote by 1 the equivalence class.

We define a dense unital *-subalgebra $A(\mathbb{G}) \subset C(\mathbb{G})$ by

$$
A(\mathbb{G})=\operatorname{span}\left\{v_{\pi_{i, j}} \mid i, j \in I_{\pi}, \pi \in \operatorname{Irr}(\mathbb{G})\right\} .
$$

It is clear that $\delta_{\mathbb{G}}(A(\mathbb{G})) \subset A(\mathbb{G}) \otimes A(\mathbb{G})$. We define the Hopf algebra structure, namely, the antipode $\kappa_{\mathbb{G}}$ and the counit $\varepsilon_{\mathbb{G}}$ on $A(\mathbb{G})$ as follows. The invertible antimultiplicative map $\kappa_{\mathbb{G}}: A(\mathbb{G}) \rightarrow A(\mathbb{G})$ is defined by

$$
\kappa_{\mathbb{G}}\left(v_{\pi_{i, j}}\right)=v_{\pi_{j, i}}^{*} \text { for } i, j \in I_{\pi}, \pi \in \operatorname{Irr}(\mathbb{G}) .
$$

The unital $*$-homomorphism $\varepsilon_{\mathbb{G}}: A(\mathbb{G}) \rightarrow \mathbb{C}$ is defined by

$$
\varepsilon_{\mathbb{G}}\left(v_{\pi_{i, j}}\right)=\delta_{i, j} \quad \text { for } i, j \in I_{\pi}, \pi \in \operatorname{Irr}(\mathbb{G}) .
$$

In fact $A(\mathbb{G})$ is a Hopf $*$-algebra, that is, $\kappa_{\mathbb{G}}\left(\kappa_{\mathbb{G}}(x)^{*}\right)^{*}=x$ holds for any $x \in$ $A(\mathbb{G})$. For any finite dimensional unitary representation $v \in B(H) \otimes A(\mathbb{G})$, we have $\left(\mathrm{id} \otimes \kappa_{\mathbb{G}}\right)(v)=v^{*}$ and $\left(\mathrm{id} \otimes \varepsilon_{\mathbb{G}}\right)(v)=1$, which follow from the complete decomposability of $v$.

We introduce the Woronowicz characters $\left\{f_{z}^{\mathbb{G}}\right\}_{z \in \mathbb{C}}$ on $A(\mathbb{G})$ [30, Theorem2.4]. The multiplicative functional $f_{z}^{\mathbb{G}}: A(\mathbb{G}) \rightarrow \mathbb{C}$ satisfies the following properties:

(i) $f_{0}^{\mathbb{G}}=\varepsilon_{\mathbb{G}}$.

(ii) For any $a \in A(\mathbb{G})$, the function $\mathbb{C} \ni z \mapsto f_{z}^{\mathbb{G}}(a) \in \mathbb{C}$ is entirely holomorphic.

(iii) $\left(f_{z_{1}}^{\mathbb{G}} \otimes f_{z_{2}}^{\mathbb{G}}\right) \circ \delta_{\mathbb{G}}=f_{z_{1}+z_{2}}^{\mathbb{G}}$ for any $z_{1}, z_{2} \in \mathbb{C}$.

(iv) For any $z \in \mathbb{C}$ and $a \in A(\mathbb{G}), f_{z}^{\mathbb{G}}(\kappa(a))=f_{-z}^{\mathbb{G}}(a), f_{z}^{\mathbb{G}}\left(a^{*}\right)=\overline{f_{-\bar{z}}^{\mathbb{G}}(a)}$.

(v) For any $a \in A(\mathbb{G}), \kappa_{\mathbb{G}}^{2}(a)=\left(f_{1}^{\mathbb{G}} \otimes \mathrm{id} \otimes f_{-1}^{\mathbb{G}}\right)\left(\left(\delta_{\mathbb{G}} \otimes \mathrm{id}\right)\left(\delta_{\mathbb{G}}(a)\right)\right)$.

(vi) For any $a, b \in A(\mathbb{G}), h_{\mathbb{G}}(a b)=h_{\mathbb{G}}\left(b\left(f_{1}^{\mathbb{G}} \otimes \mathrm{id} \otimes f_{1}^{\mathbb{G}}\right)\left(\left(\delta_{\mathbb{G}} \otimes \mathrm{id}\right)\left(\delta_{\mathbb{G}}(a)\right)\right)\right)$.

The modular automorphism group $\left\{\sigma_{t}^{h_{\mathbb{G}}}\right\}_{t \in \mathbb{R}}$ is given by

$$
\sigma_{t}^{h_{\mathbb{G}}}(x)=\left(f_{i t}^{\mathbb{G}} \otimes \mathrm{id} \otimes f_{i t}^{\mathbb{G}}\right)\left(\left(\delta_{\mathbb{G}} \otimes \mathrm{id}\right)\left(\delta_{\mathbb{G}}(x)\right)\right) \quad \text { for all } t \in \mathbb{R}, x \in A(\mathbb{G}) .
$$

We define the following map $\tau_{t}^{\mathbb{G}}: A(\mathbb{G}) \rightarrow A(\mathbb{G})$ by

$$
\tau_{t}^{\mathbb{G}}(x)=\left(f_{i t}^{\mathbb{G}} \otimes \mathrm{id} \otimes f_{-i t}^{\mathbb{G}}\right)\left(\left(\delta_{\mathbb{G}} \otimes \mathrm{id}\right)\left(\delta_{\mathbb{G}}(x)\right)\right) \quad \text { for all } t \in \mathbb{R}, x \in A(\mathbb{G}) .
$$


Then $\left\{\tau_{t}^{\mathbb{G}}\right\}_{t \in \mathbb{R}}$ is a one-parameter automorphism group on $A(\mathbb{G})$ and it is called the scaling automorphism group. Note that any element of $A(\mathbb{G})$ is analytic for $\left\{\sigma_{t}^{h_{\mathbb{G}}}\right\}_{t \in \mathbb{R}}$ and $\left\{\tau_{t}^{\mathbb{G}}\right\}_{t \in \mathbb{R}}$. Define a map $R_{\mathbb{G}}: A(\mathbb{G}) \rightarrow A(\mathbb{G})$ by

$$
R_{\mathbb{G}}(x)=\kappa_{\mathbb{G}}\left(\tau_{i / 2}^{\mathbb{G}}(x)\right) \quad \text { for } x \in A(\mathbb{G}) .
$$

Then $R_{\mathbb{G}}$ is a $*$-antiautomorphism on $A(\mathbb{G})$ with $R_{\mathbb{G}}^{2}=\mathrm{id}$. We call $R_{\mathbb{G}}$ the unitary antipode. Actually $R_{\mathbb{G}}$ commutes with $\tau_{t}^{\mathbb{G}}$ for all $t \in \mathbb{R}$, and it satisfies

$$
\kappa_{\mathbb{G}}=R_{\mathbb{G}} \circ \tau_{-i / 2}^{\mathbb{G}}=\tau_{-i / 2}^{\mathbb{G}} \circ R_{\mathbb{G}} .
$$

Since the Haar state $h_{\mathbb{G}}$ is invariant under the $*$-preserving maps $\sigma_{t}^{h_{\mathbb{G}}}, \tau_{t}^{\mathbb{G}}$ and $R_{\mathbb{G}}$, we can extend them to the maps on $C(\mathbb{G})$, moreover on $L^{\infty}(\mathbb{G})$. Then the following relations among $\delta_{\mathbb{G}}$ and them hold.

$$
\begin{array}{ll}
h_{\mathbb{G}} \circ \tau_{t}^{\mathbb{G}}=h_{\mathbb{G}}=h_{\mathbb{G}} \circ R_{\mathbb{G}}, & \delta_{\mathbb{G}} \circ \sigma_{t}^{h_{\mathbb{G}}}=\left(\sigma_{t}^{h_{\mathbb{G}}} \otimes \tau_{-t}^{\mathbb{G}}\right) \circ \delta_{\mathbb{G}}, \\
\delta_{\mathbb{G}} \circ \tau_{t}^{\mathbb{G}}=\left(\tau_{t}^{\mathbb{G}} \otimes \tau_{t}^{\mathbb{G}}\right) \circ \delta_{\mathbb{G}}, & \delta_{\mathbb{G}} \circ R_{\mathbb{G}}=\left(R_{\mathbb{G}} \otimes R_{\mathbb{G}}\right) \circ \delta_{\mathbb{G}}^{\mathrm{op}},
\end{array}
$$

where $\delta_{\mathbb{G}}^{\mathrm{op}}(x)=\delta_{\mathbb{G}}(x)_{21}$.

Let $v \in B(H) \otimes A(\mathbb{G})$ be a finite dimensional unitary representation. Set $F_{v}^{\mathbb{G}}=\left(\mathrm{id} \otimes f_{1}^{\mathbb{G}}\right)(v)$. Then $F_{v}^{\mathbb{G}}$ satisfies the following properties:

(i) $F_{v}^{\mathbb{G}}$ is a non-singular positive operator on $H$.

(ii) For any $z \in \mathbb{C}$, $\left(\mathrm{id} \otimes f_{z}^{\mathbb{G}}\right)(v)=\left(F_{v}^{\mathbb{G}}\right)^{z}$.

(iii) For any $t \in \mathbb{R},\left(\mathrm{id} \otimes \tau_{t}^{\mathbb{G}}\right)(v)=\left(\left(F_{v}^{\mathbb{G}}\right)^{i t} \otimes 1\right) v\left(\left(F_{v}^{\mathbb{G}}\right)^{-i t} \otimes 1\right)$.

(iv) For any $t \in \mathbb{R},\left(\mathrm{id} \otimes \sigma_{t}^{h_{\mathbb{G}}}\right)(v)=\left(\left(F_{v}^{\mathbb{G}}\right)^{i t} \otimes 1\right) v\left(\left(F_{v}^{\mathbb{G}}\right)^{i t} \otimes 1\right)$.

(v) Let $w \in B\left(H_{w}\right) \otimes A(\mathbb{G})$ be a finite dimensional unitary representation of $\mathbb{G}$.

If a linear map $T: H_{v} \rightarrow H_{w}$ satisfies $(T \otimes 1) v=w(T \otimes 1)$, then $T F_{v}=F_{w} T$.

For $\pi \in \operatorname{Irr}(\mathbb{G})$, we write $F_{\pi}^{\mathbb{G}}$ (or simply $F_{\pi}$ ) for $F_{v_{\pi}}^{\mathbb{G}}$. Let $\operatorname{Tr}_{\pi}$ be the nonnormalized trace on $B\left(H_{\pi}\right)$. Set $D_{\pi}=\operatorname{Tr}_{\pi}\left(F_{\pi}\right)$. Then we have the orthogonal relations,

$$
h_{\mathbb{G}}\left(v_{\pi_{i, j}} v_{\rho_{k, \ell}}^{*}\right)=D_{\pi}^{-1} F_{\pi_{j, j}} \delta_{\pi, \rho} \delta_{i, k} \delta_{j, \ell}, \quad h_{\mathbb{G}}\left(v_{\pi_{i, j}}^{*} v_{\rho_{k, \ell}}\right)=D_{\pi}^{-1} F_{\pi_{i, i}}^{-1} \delta_{\pi, \rho} \delta_{i, k} \delta_{j, \ell} .
$$

We decompose $W_{\mathbb{G}}$ and $V_{\mathbb{G}}$ into irreducible representations. For $\pi \in \operatorname{Irr}(\mathbb{G})$, we define two systems of matrix units $\left\{e_{\pi_{i, j}}\right\}_{i, j \in I_{\pi}}$ and $\left\{f_{\pi_{i, j}}\right\}_{i, j \in I_{\pi}}$ in $B\left(L^{2}(\mathbb{G})\right)$ by

$$
\begin{aligned}
& e_{\pi_{i, j}}\left(v_{\rho_{k, \ell}} \hat{1}_{h}\right)=\delta_{\pi, \rho} \delta_{j, \ell} v_{\rho_{k, i}} \hat{1}_{h} \quad \text { for } k, \ell \in I_{\rho}, \rho \in \operatorname{Irr}(\mathbb{G}), \\
& f_{\pi_{i, j}}\left(v_{\rho_{k, \ell}}^{*} \hat{1}_{h}\right)=\delta_{\pi, \rho} \delta_{j, k} v_{\rho_{i, \ell}}^{*} \hat{1}_{h} \quad \text { for } k, \ell \in I_{\rho}, \rho \in \operatorname{Irr}(\mathbb{G}) .
\end{aligned}
$$

Then we have

$$
W_{\mathbb{G}}=\sum_{\pi \in \operatorname{Irr}(\mathbb{G})} \sum_{i, j \in I_{\pi}} v_{\pi_{i, j}} \otimes f_{\pi_{i, j}}, \quad V_{\mathbb{G}}=\sum_{\pi \in \operatorname{Irr}(\mathbb{G})} \sum_{i, j \in I_{\pi}} e_{\pi_{i, j}} \otimes v_{\pi_{i, j}} .
$$

Setting $\pi=1$ at (2.8), we have

$$
e_{\mathbf{1}}\left(x \hat{1}_{h}\right)=h_{\mathbb{G}}(x) \hat{1}_{h} \quad \text { for } x \in L^{\infty}(\mathbb{G}) .
$$

The projection $e_{\mathbf{1}}=f_{\mathbf{1}}$ is minimal in $B\left(L^{2}(\mathbb{G})\right)$.

We will need some relations among $W_{\mathbb{G}}, V_{\mathbb{G}}$ and the modular objects of $L^{\infty}(\mathbb{G})$. Let $\Delta_{h_{\mathbb{G}}}$ and $J_{\mathbb{G}}$ be the modular operator and the modular conjugation of $h_{\mathbb{G}}$. We 
define a conjugate unitary $\hat{J}_{\mathbb{G}}$ by $\hat{J}_{\mathbb{G}}\left(x \hat{1}_{h}\right)=R_{\mathbb{G}}\left(x^{*}\right) \hat{1}_{h}$ for $x \in C(\mathbb{G})$. We set a unitary $U_{\mathbb{G}}=J_{\mathbb{G}} \hat{J}_{\mathbb{G}}=\hat{J}_{\mathbb{G}} J_{\mathbb{G}}$. The implementing unitary $P_{\mathbb{G}}^{i t}$ for $\tau_{t}^{\mathbb{G}}$ is defined by $P_{\mathbb{G}}^{i t}\left(x \hat{1}_{h}\right)=\tau_{t}^{\mathbb{G}}(x) \hat{1}_{h}$ for $t \in \mathbb{R}$ and $x \in L^{\infty}(\mathbb{G})$. Then the following equalities are directly deduced from (2.1), (2.2), (2.6) and (2.7).

$$
\begin{array}{ll}
W_{\mathbb{G}}=\left(\hat{J}_{\mathbb{G}} \otimes J_{\mathbb{G}}\right) W_{\mathbb{G}}^{*}\left(\hat{J}_{\mathbb{G}} \otimes J_{\mathbb{G}}\right), & V_{\mathbb{G}}=\left(J_{\mathbb{G}} \otimes \hat{J}_{\mathbb{G}}\right) V_{\mathbb{G}}^{*}\left(J_{\mathbb{G}} \otimes \hat{J}_{\mathbb{G}}\right), \\
W_{\mathbb{G}}=\left(P_{\mathbb{G}}^{i t} \otimes P_{\mathbb{G}}^{i t}\right) W_{\mathbb{G}}\left(P_{\mathbb{G}}^{-i t} \otimes P_{\mathbb{G}}^{-i t}\right), & V_{\mathbb{G}}=\left(P_{\mathbb{G}}^{i t} \otimes P_{\mathbb{G}}^{i t}\right) V_{\mathbb{G}}\left(P_{\mathbb{G}}^{-i t} \otimes P_{\mathbb{G}}^{-i t}\right), \\
W_{\mathbb{G}}=\left(P_{\mathbb{G}}^{i t} \otimes \Delta_{h_{\mathbb{G}}}^{i t}\right) W_{\mathbb{G}}\left(P_{\mathbb{G}}^{-i t} \otimes \Delta_{h_{\mathbb{G}}}^{-i t}\right), & V_{\mathbb{G}}=\left(\Delta_{h_{\mathbb{G}}}^{i t} \otimes P_{\mathbb{G}}^{-i t}\right) V_{\mathbb{G}}\left(\Delta_{h_{\mathbb{G}}}^{-i t} \otimes P_{\mathbb{G}}^{i t}\right), \\
W_{\mathbb{G}}=\left(\hat{J}_{\mathbb{G}} \otimes \hat{J}_{\mathbb{G}}\right)\left(V_{\mathbb{G}}\right)_{21}^{*}\left(\hat{J}_{\mathbb{G}} \otimes \hat{J}_{\mathbb{G}}\right) . &
\end{array}
$$

We denote by $L^{\infty}(\mathbb{G})_{*}^{\text {fin }}$ the set of $\omega \in L^{\infty}(\mathbb{G})_{*}$ which satisfies $\omega\left(v_{\pi_{i, j}}\right)=0$, $i, j \in I_{\pi}$ all but finite elements $\pi \in \operatorname{Irr}(\mathbb{G})$. We simply write symbols by omitting $\mathbb{G}$, if no confusion arise. For example, we write $\delta$ for $\delta_{\mathbb{G}}$.

\subsection{Discrete quantum groups.}

The notions of a (dual) discrete quantum group have been studied in many papers, for example, [6], [16], [27], [29] and [31]. They have described essentially the same object. In this paper, we use a von Neumann algebraic quantum group presented in [16].

Definition 2.2. A discrete quantum group is a quintuplet $(M, \Delta, \varphi, \psi, \varepsilon)$ which satisfies the following conditions:

(1) $M$ is a separable von Neumann algebra.

(2) $\Delta: M \rightarrow M \otimes M$ is a coproduct, that is, it is a faithful normal unital $*$-homomorphism satisfying the coassociativity condition,

$$
(\Delta \otimes \mathrm{id}) \circ \Delta=(\mathrm{id} \otimes \Delta) \circ \Delta \text {. }
$$

(3) $\varphi$ is a faithful normal semifinite weight on $M$ satisfying the left invariance,

$$
\varphi((\omega \otimes \mathrm{id})(\Delta(x)))=\omega(1) \varphi(x) \quad \text { for all } \omega \in M_{*}^{+}, x \in m_{\varphi}^{+} .
$$

(4) $\psi$ is a faithful normal semifinite weight on $M$ satisfying the right invariance,

$$
\psi((\mathrm{id} \otimes \omega)(\Delta(x)))=\omega(1) \psi(x) \quad \text { for all } \omega \in M_{*}^{+}, x \in m_{\psi}^{+} .
$$

(5) $\varepsilon$ is a normal counit, that is, it is a normal character on $M$ satisfying

$$
(\varepsilon \otimes \mathrm{id}) \circ \Delta=\mathrm{id}=(\mathrm{id} \otimes \varepsilon) \circ \Delta .
$$

In fact, those weights $\varphi, \psi$ are uniquely determined up to scalar multiplications and the counit $\varepsilon$ is unique. From now, we simply write $(M, \Delta)$ for $(M, \Delta, \varphi, \psi, \varepsilon)$ by omitting $\varphi, \psi$ and $\varepsilon$ once they are given.

For a compact quantum group $\mathbb{G}$, we construct the dual discrete quantum group as follows (see for example [1], [16]). We define the left group algebra and right group algebra by

$$
L(\mathbb{G})=\overline{\left\{(\omega \otimes \mathrm{id})\left(W_{\mathbb{G}}\right) \mid \omega \in L^{\infty}(\mathbb{G})_{*}\right\}^{\mathrm{w}}}{ }_{6}^{\mathrm{w}}, R(\mathbb{G})=\overline{\left\{(\mathrm{id} \otimes \omega)\left(V_{\mathbb{G}}\right) \mid \omega \in L^{\infty}(\mathbb{G})_{*}\right\}}{ }^{\mathrm{w}} .
$$


By $(2.10), L(\mathbb{G})$ and $R(\mathbb{G})$ are generated by $\left\{f_{\pi_{i, j}}\right\}$ and $\left\{e_{\pi_{i, j}}\right\}$, respectively. Hence they are isomorphic to the von Neumann algebra direct sum of the matrix algebras $\left\{B\left(H_{\pi}\right)\right\}_{\pi \in \operatorname{Irr}(\mathbb{G})}$. Set

$$
L(\mathbb{G})^{\mathrm{fin}}=\left\{(\omega \otimes \mathrm{id})\left(W_{\mathbb{G}}\right) \mid \omega \in L^{\infty}(\mathbb{G})_{*}^{\mathrm{fin}}\right\}, R(\mathbb{G})^{\mathrm{fin}}=\left\{(\mathrm{id} \otimes \omega)\left(V_{\mathbb{G}}\right) \mid \omega \in L^{\infty}(\mathbb{G})_{*}^{\mathrm{fin}^{\mathrm{fi}}}\right\} .
$$

They are algebraic direct sum of the matrix algebras. We note the commutant property $L(\mathbb{G})^{\prime}=R(\mathbb{G})=\hat{J} L(\mathbb{G}) \hat{J}$. We define a minimal central projection corresponding to $\pi \in \operatorname{Irr}(\mathbb{G})$ by $1_{\pi}=\sum_{i \in I_{\pi}} f_{\pi_{i, i}}$. In particular, $e_{\mathbf{1}}=1_{\mathbf{1}}$. We denote by $L(\mathbb{G})_{*}^{\mathrm{fin}}$ the subset of $L(\mathbb{G})_{*}$ which consists of $\omega \in L(\mathbb{G})_{*}$ such that $\omega\left(f_{\pi_{i, j}}\right)=0, i, j \in I_{\pi}$ all but finite elements $\pi \in \operatorname{Irr}(\mathbb{G})$.

We prepare the coproducts $\Delta_{L}$ and $\Delta_{R}$ defined by

$$
\begin{array}{ll}
\Delta_{L}(x)=W_{\mathbb{G}}(x \otimes 1) W_{\mathbb{G}}^{*} & \text { for } x \in L(\mathbb{G}), \\
\Delta_{R}(x)=V_{\mathbb{G}}^{*}(1 \otimes x) V_{\mathbb{G}} & \text { for } x \in R(\mathbb{G}) .
\end{array}
$$

We define the normal counit $\hat{\varepsilon}: L(\mathbb{G}) \rightarrow \mathbb{C}$ by

$$
\hat{\varepsilon}(x) e_{\mathbf{1}}=x e_{\mathbf{1}} \quad \text { for } x \in L(\mathbb{G}) .
$$

In fact, there exist left, right invariant weights on $L(\mathbb{G})$ and $R(\mathbb{G})[16$, and they are discrete quantum groups. Note that every discrete quantum group arises as the left (right) group algebra of a compact quantum group (see [1, 16] for duality theory). In this paper, we use the symbol $\widehat{\mathbb{G}}$ for the discrete quantum group $\left(R(\mathbb{G}), \Delta_{R}\right)$. We simply write $\Delta_{\mathbb{G}}($ or $\Delta)$ for $\Delta_{L}$ and $\Delta_{R}$ when it is not ambiguous.

We define a positive operator $F$ affiliated with $L(\mathbb{G})$ by

$$
F=\sum_{\pi \in \operatorname{Irr}(\mathbb{G})} \sum_{i \in I_{\pi}} F_{\pi_{i, i}} f_{\pi_{i, i}} .
$$

Using (2.9), for all $t \in \mathbb{R}$ and $x \in A(\mathbb{G})$ we have

$$
F^{i t}\left(x \hat{1}_{h}\right)=\left(f_{-i t} \otimes \mathrm{id}\right)(\delta(x)) \hat{1}_{h}, \quad \hat{J} F^{i t} \hat{J}\left(x \hat{1}_{h}\right)=\left(\mathrm{id} \otimes f_{i t}\right)(\delta(x)) \hat{1}_{h} .
$$

Then we have

$$
\Delta_{h}^{i t}=F^{-i t} \hat{J} F^{i t} \hat{J}, \quad P^{i t}=F^{-i t} \hat{J} F^{-i t} \hat{J} .
$$

The antipode $\hat{S}$ on $L(\mathbb{G})$ is defined as follows. Since the map $L^{\infty}(\mathbb{G})_{*} \ni \omega \mapsto$ $(\omega \otimes \mathrm{id})\left(W_{\mathbb{G}}\right) \in L^{\infty}(\mathbb{G})$ is injective, we can define an invertible antimultiplicative map $\hat{S}: L(\mathbb{G})^{\mathrm{fin}} \rightarrow L(\mathbb{G})^{\mathrm{fin}}$ by

$$
\hat{S}\left((\omega \otimes \mathrm{id})\left(W_{\mathbb{G}}\right)\right)=(\omega \otimes \mathrm{id})\left(W_{\mathbb{G}}^{*}\right) \text { for all } \omega \in L^{\infty}(\mathbb{G})_{*}^{\mathrm{fin}} .
$$

We define the unitary antipode $\hat{R}$ and the scaling automorphism group $\left\{\hat{\tau}_{t}\right\}_{t \in \mathbb{R}}$ on $L(\mathbb{G})$ by

$$
\hat{R}(x)=J x^{*} J, \quad \hat{\tau}_{t}(x)=F^{i t} x F^{-i t} \quad \text { for } x \in L(\mathbb{G}), t \in \mathbb{R} .
$$

Using (2.11), (2.12) and (2.15), we have $\hat{S}=\hat{R} \circ \hat{\tau}_{-i / 2}$ on $L(\mathbb{G})^{\text {fin }}$. The relations among $\Delta, \hat{R}$ and $\hat{\tau}$ are as follows.

$$
\Delta \circ \hat{R}=(\hat{R} \otimes \hat{R}) \circ \Delta^{\mathrm{op}}, \quad \Delta \circ \hat{\tau}_{t}=\left(\hat{\tau}_{t} \otimes \hat{\tau}_{t}\right) \circ \Delta .
$$


Take $\bar{\pi} \in \operatorname{Irr}(\mathbb{G})$ satisfying $\hat{S}\left(1_{\pi}\right)=1_{\bar{\pi}}$. Let $\mu: L(\mathbb{G})^{\text {fin }} \otimes L(\mathbb{G})^{\text {fin }} \rightarrow L(\mathbb{G})^{\text {fin }}$ be the multiplication map. We prove the well-known results for readers' convenience.

Lemma 2.3. For any $x \in L(\mathbb{G})$ and $\pi \in \operatorname{Irr}(\mathbb{G})$, one has

$$
\mu\left((\mathrm{id} \otimes \hat{S})\left({ }_{\bar{\pi}} \Delta_{\pi}(x)\right)\right)=\hat{\varepsilon}(x) 1_{\bar{\pi}}=\mu\left((\hat{S} \otimes \mathrm{id})\left({ }_{\pi} \Delta_{\bar{\pi}}(x)\right)\right),
$$

where ${ }_{\pi} \Delta_{\bar{\pi}}(x)=\Delta(x)\left(1_{\pi} \otimes 1_{\bar{\pi}}\right)$.

Proof. We prove $\mu\left((\operatorname{id} \otimes \hat{S})\left(_{\bar{\pi}} \Delta_{\pi}(x)\right)\right)=\hat{\varepsilon}(x) 1_{\bar{\pi}}$. Since ${ }_{\pi} \Delta_{\bar{\pi}}\left(1_{\rho}\right)$ is non-zero for finite elements $\rho \in \operatorname{Irr}(\mathbb{G})$, we may assume that $x=(\omega \otimes \mathrm{id})\left(W_{\mathbb{G}}\right), \omega \in L^{\infty}(\mathbb{G})_{*}^{\mathrm{fin}}$. Then we have

$$
\begin{aligned}
(\mathrm{id} \otimes \hat{S}){\left({ }_{\pi} \Delta_{\pi}(x)\right)} & =(\mathrm{id} \otimes \hat{S})\left({ }_{\pi} \Delta_{\pi}\left((\omega \otimes \mathrm{id})\left(W_{\mathbb{G}}\right)\right)\right) \\
& =(\mathrm{id} \otimes \hat{S})\left((\omega \otimes \mathrm{id} \otimes \mathrm{id})\left(\left(\mathrm{id} \otimes_{\bar{\pi}} \Delta_{\pi}\right)\left(W_{\mathbb{G}}\right)\right)\right) \\
& =(\mathrm{id} \otimes \hat{S})\left((\omega \otimes \mathrm{id} \otimes \mathrm{id})\left(\left(W_{\mathbb{G}}\right)_{12}\left(W_{\mathbb{G}}\right)_{13}\left(1 \otimes 1_{\bar{\pi}} \otimes 1_{\pi}\right)\right)\right) \\
& =(\omega \otimes \mathrm{id} \otimes \mathrm{id})\left(\left(W_{\mathbb{G}}\right)_{12}\left(W_{\mathbb{G}}\right)_{13}^{*}\left(1 \otimes 1_{\bar{\pi}} \otimes 1_{\bar{\pi}}\right)\right) .
\end{aligned}
$$

Hence

$$
\begin{aligned}
\mu\left((\operatorname{id} \otimes \hat{S})\left(_{\bar{\pi}} \Delta_{\pi}(x)\right)\right) & =\mu\left((\omega \otimes \mathrm{id} \otimes \mathrm{id})\left(\left(W_{\mathbb{G}}\right)_{12}\left(W_{\mathbb{G}}\right)_{13}^{*}\left(1 \otimes 1_{\bar{\pi}} \otimes 1_{\bar{\pi}}\right)\right)\right) \\
& =(\omega \otimes \mathrm{id})\left(W_{\mathbb{G}} W_{\mathbb{G}}^{*}\left(1 \otimes 1_{\bar{\pi}}\right)\right) \\
& =\omega(1) 1_{\bar{\pi}}=\hat{\varepsilon}(x) 1_{\bar{\pi}} .
\end{aligned}
$$

Similarly we can prove $\mu\left((\hat{S} \otimes \mathrm{id})\left({ }_{\pi} \Delta_{\bar{\pi}}(x)\right)\right)=\hat{\varepsilon}(x) 1_{\bar{\pi}}$.

Lemma 2.4. Let $K$ be a Hilbert space and $\mathcal{V} \in B(K) \otimes L(\mathbb{G})$ a unitary representation of $(L(\mathbb{G}), \Delta)$, that is, $\mathcal{V}$ is a unitary satisfying $(\mathrm{id} \otimes \Delta)(\mathcal{V})=\mathcal{V}_{12} \mathcal{V}_{13}$. Then for any $\pi \in \operatorname{Irr}(\mathbb{G})$, one has

$$
(\operatorname{id} \otimes \hat{S})\left(\mathcal{V}_{\pi}\right)=\mathcal{V}_{\bar{\pi}}^{*}
$$

where $\mathcal{V}_{\pi}=\mathcal{V}\left(1 \otimes 1_{\pi}\right)$.

Proof. Set $x=(\omega \otimes \mathrm{id})(\mathcal{V}) \in L(\mathbb{G}), \omega \in B(K)_{*}$. For any $\pi \in \operatorname{Irr}(\mathbb{G})$, we have

$$
\begin{aligned}
\left.(\mathrm{id} \otimes \hat{S}){ }_{(\bar{\pi}} \Delta_{\pi}(x)\right) & =(\mathrm{id} \otimes \hat{S})\left((\omega \otimes \mathrm{id} \otimes \mathrm{id})\left(\left(\mathrm{id} \otimes_{\bar{\pi}} \Delta_{\pi}\right)(\mathcal{V})\right)\right) \\
& =(\omega \otimes \mathrm{id} \otimes \hat{S})\left(\left(\mathcal{V}_{\bar{\pi}}\right)_{12}\left(\mathcal{V}_{\pi}\right)_{13}\right) \\
& =(\omega \otimes \mathrm{id} \otimes \mathrm{id})\left(\left(\mathcal{V}_{\bar{\pi}}\right)_{12}(\mathrm{id} \otimes \hat{S})\left(\mathcal{V}_{\pi}\right)_{13}\right)
\end{aligned}
$$

By the previous lemma, we have $\left.\mu\left((\operatorname{id} \otimes \hat{S}){ }_{\pi} \Delta_{\pi}(x)\right)\right)=\hat{\varepsilon}(x) 1$. This implies $(\omega \otimes$ id $)\left(\mathcal{V}_{\bar{\pi}}(\mathrm{id} \otimes \hat{S})\left(\mathcal{V}_{\pi}\right)\right)=\omega(1)$ for any $\omega \in B(K)_{*}$. Hence $(\operatorname{id} \otimes \hat{S})\left(\mathcal{V}_{\pi}\right)=\mathcal{V}_{\bar{\pi}}^{*}$.

\subsection{Amenability.}

We recall the notion of amenability of a discrete quantum group. For a detail of the theory, readers are referred to [2], [3], [4], 22] and references therein. A discrete quantum group $(M, \Delta)$ is said to be amenable if there exists a state $m \in M^{*}$ such that $m((\omega \otimes \mathrm{id})(\Delta(x)))=\omega(1) m(x)$ for all $\omega \in M_{*}$ and $x \in M$. The state $m$ is called a left invariant mean. It is known that $\left(L(\mathbb{G}), \Delta_{L}\right)$ is 
amenable if and only if $\left(R(\mathbb{G}), \Delta_{R}\right)$ is amenable. If it is the case, we say that $\mathbb{G}$ is co-amenable. Note that the counit $\varepsilon_{\mathbb{G}}$ is norm-bounded if and only if $\mathbb{G}$ is coamenable [4, Theorem 4.7], [22, Theorem 3.8]. The amenability is also equivalent to the universality of $C(\mathbb{G})$, that is, for any $C^{*}$-algebra $B$, any $*$-homomorphism $\sigma: A(\mathbb{G}) \rightarrow B$ extends to a $*$-homomorphism $\sigma: C(\mathbb{G}) \rightarrow B$ [3, Theorem 3.6].

\subsection{Right $\mathbb{G}$-action $\alpha$ and left $\widehat{\mathbb{G}}$-action $\beta$.}

We prepare two maps $\alpha: B\left(L^{2}(\mathbb{G})\right) \rightarrow B\left(L^{2}(\mathbb{G})\right) \otimes L^{\infty}(\mathbb{G})$ and $\beta: B\left(L^{2}(\mathbb{G})\right) \rightarrow$ $R(\mathbb{G}) \otimes B\left(L^{2}(\mathbb{G})\right)$ which will be frequently used in our study. A map $\alpha$ is defined by

$$
\alpha(x)=V_{\mathbb{G}}(x \otimes 1) V_{\mathbb{G}}^{*} \text { for } x \in B\left(L^{2}(\mathbb{G})\right) .
$$

Then $\alpha$ is a right action of $\mathbb{G}$ on $B\left(L^{2}(\mathbb{G})\right)$, that is, $(\alpha \otimes \mathrm{id}) \circ \alpha=\left(\mathrm{id} \otimes \delta_{\mathbb{G}}\right) \circ \alpha$ holds. The conditional expectation $E_{\alpha}=(\mathrm{id} \otimes h) \circ \alpha$ maps $B\left(L^{2}(\mathbb{G})\right)$ onto $L(\mathbb{G})=$ $\left\{x \in B\left(L^{2}(\mathbb{G})\right) \mid \alpha(x)=x \otimes 1\right\}$. The other one, $\beta$ is defined by

$$
\beta(x)=V_{\mathbb{G}}^{*}(1 \otimes x) V_{\mathbb{G}} \quad \text { for } x \in B\left(L^{2}(\mathbb{G})\right) .
$$

Then $\beta$ is a left action of $\widehat{\mathbb{G}}$ on $B\left(L^{2}(\mathbb{G})\right)$, that is, $(\mathrm{id} \otimes \beta) \circ \beta=\left(\Delta_{R} \otimes\right.$ id $) \circ \beta$ holds. We also call $\beta$ a coaction of $\mathbb{G}$. Note that both the actions $\alpha, \beta$ preserve not only $L^{\infty}(\mathbb{G})$ but also $R(\mathbb{G})$.

\subsection{Right coideals.}

We introduce the notion of a right coideal. Our basic references for theory of right coideals are [11] and [23].

Definition 2.5. Let $\mathbb{G}$ be a compact quantum group. Let $B \subset L^{\infty}(\mathbb{G})$ be a von Neumann subalgebra. We say that $B$ is a right coideal if $\delta(B) \subset B \otimes L^{\infty}(\mathbb{G})$.

A left coideal in $L(\mathbb{G})$ is similarly defined. For a right coideal $B \subset L^{\infty}(\mathbb{G})$ and a left coideal $C \subset L(\mathbb{G})$, we define

$$
\widehat{B}=B^{\prime} \cap L(\mathbb{G}), \quad \widehat{C}=C^{\prime} \cap L^{\infty}(\mathbb{G}) .
$$

We prove a quantum group version of [11, Theorem 4.6] as follows.

Lemma 2.6. Let $\mathbb{G}$ be a compact quantum group. Let $B \subset L^{\infty}(\mathbb{G})$ be a right coideal and $C \subset L(\mathbb{G})$ a left coideal.

(1) $\widehat{B} \subset L(\mathbb{G})$ is a left coideal and $\widehat{C} \subset L^{\infty}(\mathbb{G})$ is a right coideal.

(2) The map $B \mapsto \widehat{B}$ is a lattice anti-isomorphism between the set of right coideal of $L^{\infty}(\mathbb{G})$ and left coideal of $L(\mathbb{G})$. The inverse map is given by $C \mapsto \widehat{C}$.

Proof. (1). It is similarly proved as in [11, Theorem 4.6].

(2). First we show that the map $C \mapsto \widehat{C}$ is injective. Let $C \subset L(\mathbb{G})$ be a left coideal. Then $\widehat{C}^{\prime}=\left(C^{\prime} \cap L^{\infty}(\mathbb{G})\right)^{\prime}=C \vee L^{\infty}(\mathbb{G})^{\prime}$. We can adapt the proof of [11, Theorem 4.6 (ii)] to deduce $\widehat{C}^{\prime}={\overline{C L^{\infty}\left(\mathbb{G}^{\prime}\right.}}^{\mathrm{w}}$. Recall the right $\mathbb{G}$-action $\alpha$ on $B\left(L^{2}(\mathbb{G})\right)$ and the conditional expectation $E_{\alpha}: B\left(L^{2}(\mathbb{G})\right) \rightarrow L(\mathbb{G})$. We apply $E_{\alpha}$ to $J \widehat{C}^{\prime} J={\overline{J C J L^{\infty}(\mathbb{G})}}^{\mathrm{w}}$. Since $J C J \subset J L(\mathbb{G}) J \subset L(\mathbb{G})$ and the restriction 
of $E_{\alpha}$ to $L^{\infty}(\mathbb{G})$ is the Haar state $h$, we have $E_{\alpha}\left(J \widehat{C}^{\prime} J\right)=J C J$. Hence the map $C \mapsto \widehat{C}$ is injective. Set $B=\widehat{C}$. Then $\widehat{B}^{\prime} \cap L^{\infty}(\mathbb{G})=\widehat{C}$ holds. The injectivity of the map $C \mapsto \widehat{C}$ yields $\widehat{B}=C$.

Next we show that the map $B \mapsto \widehat{B}$ is injective. Let $B_{i} \subset L^{\infty}(\mathbb{G}), i=1,2$ be right coideals. Then $\widehat{B}_{i}^{\prime}=\left(B_{i}^{\prime} \cap L(\mathbb{G})\right)^{\prime}=B_{i} \vee R(\mathbb{G})$. Let $B_{i} \rtimes_{\delta} \mathbb{G}=\delta\left(B_{i}\right) \vee \mathbb{C} \otimes$ $R(\mathbb{G})$ be the crossed product. Then we have $W_{\mathbb{G}}\left(B_{i} \rtimes_{\delta} \mathbb{G}\right) W_{\mathbb{G}}^{*}=\mathbb{C} \otimes\left(B_{i} \vee R(\mathbb{G})\right)=$ $\mathbb{C} \otimes \widehat{B}_{i}^{\prime}$. Hence $\widehat{B}_{1}=\widehat{B}_{2}$ if and only if $B_{1} \rtimes_{\delta} \mathbb{G}=B_{2} \rtimes_{\delta} \mathbb{G}$. Then the biduality theorem (see [1, Théorème 7.5] or [24, Theorem 2.6]) implies that it is equivalent to $B_{1} \otimes B\left(L^{2}(\mathbb{G})\right)=B_{2} \otimes B\left(L^{2}(\mathbb{G})\right)$, and $B_{1}=B_{2}$. Hence the map $B \mapsto \widehat{B}$ is injective.

\subsection{Quantum subgroups.}

For the definition of quantum subgroups, we follow that of [19], in which matrix pseudogroups [28] are treated.

Definition 2.7. Let $\mathbb{G}$ and $\mathbb{H}$ be compact quantum groups.

(1) Suppose that there exists a surjective $*$-homomorphism $r_{\mathbb{H}}: A(\mathbb{G}) \rightarrow A(\mathbb{H})$ such that $\delta_{\mathbb{H}} \circ r_{\mathbb{H}}=\left(r_{\mathbb{H}} \otimes r_{\mathbb{H}}\right) \circ \delta_{\mathbb{G}}$. Then we say that the pair $\left\{\mathbb{H}, r_{\mathbb{H}}\right\}$ is an algebraic quantum subgroup of $\mathbb{G}$.

(2) Suppose that there exists a surjective $*$-homomorphism $r_{\mathbb{H}}: C(\mathbb{G}) \rightarrow C(\mathbb{H})$ such that $\delta_{\mathbb{H}} \circ r_{\mathbb{H}}=\left(r_{\mathbb{H}} \otimes r_{\mathbb{H}}\right) \circ \delta_{\mathbb{G}}$. Then we say that the pair $\left\{\mathbb{H}, r_{\mathbb{H}}\right\}$ is a quantum subgroup of $\mathbb{G}$.

In the above cases, the map $r_{\mathbb{H}}$ is called a restriction map. We present basic properties on (algebraic) quantum subgroups in the following lemmas. Those are probably well-known for specialists, but we prove them for the sake of readers' convenience.

Lemma 2.8. Let $\mathbb{G}$ and $\mathbb{H}$ be compact quantum groups.

(1) If $\left\{\mathbb{H}, r_{\mathbb{H}}\right\}$ is a quantum subgroup of $\mathbb{G}$, then it is an algebraic quantum subgroup of $\mathbb{G}$.

(2) Suppose $\mathbb{G}$ is co-amenable. Then any algebraic quantum subgroup of $\mathbb{G}$ is naturally regarded as a quantum subgroup.

Proof. (1). Let $\pi \in \operatorname{Irr}(\mathbb{G})$. Then $\left(\mathrm{id} \otimes r_{\mathbb{H}}\right)\left(v_{\pi}\right) \in B\left(H_{\pi}\right) \otimes C(\mathbb{H})$ is a finite dimensional unitary representation of $\mathbb{H}$. Hence it is a finite direct sum of irreducible representations of $\mathbb{H}$. This implies $\left(\mathrm{id} \otimes r_{\mathbb{H}}\right)\left(v_{\pi}\right) \in B\left(H_{\pi}\right) \otimes A(\mathbb{H})$. Hence $r_{\mathbb{H}}(A(\mathbb{G})) \subset A(\mathbb{H})$. Next we show the converse inclusion. Take any $\rho \in \operatorname{Irr}(\mathbb{H})$. Let $w_{\rho} \in B\left(K_{\rho}\right) \otimes A(\mathbb{H})$ be a corresponding irreducible unitary representation. Consider the bounded linear map $\theta: C(\mathbb{G}) \rightarrow B\left(K_{\rho}\right)$ defined by $\theta(x)=\left(\mathrm{id} \otimes h_{\mathbb{H}}\right)\left(w_{\rho}^{*}\left(1 \otimes r_{\mathbb{H}}(x)\right)\right)$. Since $r_{\mathbb{H}}$ is surjective, $\theta$ is a non-zero map. By density of $A(\mathbb{G}) \subset C(\mathbb{G})$, There exists $\pi \in \operatorname{Irr}(\mathbb{G})$ such that $(\mathrm{id} \otimes \theta)\left(v_{\pi}\right) \neq 0$. This shows that the unitary representation $\left(\mathrm{id} \otimes r_{\mathbb{H}}\right)\left(v_{\pi}\right)$ contains $w_{\rho}$. Hence all the entries of $w_{\rho}$ are contained in $r_{\mathbb{H}}(A(\mathbb{G}))$, and $A(\mathbb{H}) \subset r_{\mathbb{H}}(A(\mathbb{G})$ ).

$(2)$. Let $r_{\mathbb{H}}: A(\mathbb{G}) \rightarrow A(\mathbb{H})$ be a restriction map. Since $C(\mathbb{G})$ is universal, the map extends to $r_{\mathbb{H}}: C(\mathbb{G}) \rightarrow C(\mathbb{H})$. The image contains a total subspace $A(\mathbb{H})$ in 
$C(\mathbb{H})$, and $r_{\mathbb{H}}$ is surjective. By continuity of $r_{\mathbb{H}}$, the relation $\delta_{\mathbb{H}} \circ r_{\mathbb{H}}=\left(r_{\mathbb{H}} \otimes r_{\mathbb{H}}\right) \circ \delta_{\mathbb{G}}$ holds on $C(\mathbb{G})$. Hence $\left\{\mathbb{H}, r_{\mathbb{H}}\right\}$ is a quantum subgroup of $\mathbb{G}$.

Lemma 2.9. Let $\left\{\mathbb{H}, r_{\mathbb{H}}\right\}$ be an algebraic quantum subgroup of $\mathbb{G}$. On $A(\mathbb{G})$,

(1) $\varepsilon_{\mathbb{H}} \circ r_{\mathbb{H}}=\varepsilon_{\mathbb{G}}$

(2) $\tau_{t}^{\mathbb{H}} \circ r_{\mathbb{H}}=r_{\mathbb{H}} \circ \tau_{t}^{\mathbb{G}}$ for all $t \in \mathbb{R}$,

(3) $R_{\mathbb{H}} \circ r_{\mathbb{H}}=r_{\mathbb{H}} \circ R_{\mathbb{G}}$.

Proof. (1). Take any $\pi \in \operatorname{Irr}(\mathbb{G})$. Since $\left(\mathrm{id} \otimes r_{\mathbb{H}}\right)\left(v_{\pi}\right)$ is a unitary representation of $\mathbb{H},\left(\mathrm{id} \otimes \varepsilon_{\mathbb{H}} \circ r_{\mathbb{H}}\right)\left(v_{\pi}\right)=1=\left(\mathrm{id} \otimes \varepsilon_{\mathbb{G}}\right)\left(v_{\pi}\right)$. This implies $\varepsilon_{\mathbb{H}} \circ r_{\mathbb{H}}=\varepsilon_{\mathbb{G}}$.

$(2)$. Take any $\pi \in \operatorname{Irr}(\mathbb{G})$. Since $\left(\mathrm{id} \otimes r_{\mathbb{H}}\right)\left(v_{\pi}\right)$ is a unitary representation of $\mathbb{H}$, $\left(\mathrm{id} \otimes \kappa_{\mathbb{H}} \circ r_{\mathbb{H}}\right)\left(v_{\pi}\right)=\left(\mathrm{id} \otimes r_{\mathbb{H}}\right)\left(v_{\pi}\right)^{*}=\left(\mathrm{id} \otimes r_{\mathbb{H}} \circ \kappa_{\mathbb{G}}\right)\left(v_{\pi}\right)$. This implies $r_{\mathbb{H}} \circ \kappa_{\mathbb{G}}=$ $\kappa_{\mathbb{H}} \circ r_{\mathbb{H}}$. Since $\kappa_{\mathbb{G}}^{2}=\tau_{-i}^{\mathbb{G}}$ and $\kappa_{\mathbb{H}}^{2}=\tau_{-i}^{\mathbb{H}}$, we have $r_{\mathbb{H}} \circ \tau_{-i}^{\mathbb{G}}=\tau_{-i}^{\mathbb{H}} \circ r_{\mathbb{H}}$. Let $\pi \in \operatorname{Irr}(\mathbb{G})$ and set $w=\left(\mathrm{id} \otimes r_{\mathbb{H}}\right)\left(v_{\pi}\right)$. Then we have

$$
\begin{aligned}
\left(F_{\pi}^{\mathbb{G}} \otimes 1\right) w\left(\left(F_{\pi}^{\mathbb{G}}\right)^{-1} \otimes 1\right) & =\left(\mathrm{id} \otimes r_{\mathbb{H}}\right)\left(\left(F_{\pi}^{\mathbb{G}} \otimes 1\right) v_{\pi}\left(\left(F_{\pi}^{\mathbb{G}}\right)^{-1} \otimes 1\right)\right)=\left(\mathrm{id} \otimes r_{\mathbb{H}} \circ \tau_{-i}^{\mathbb{G}}\right)\left(v_{\pi}\right) \\
& =\left(\mathrm{id} \otimes \tau_{-i}^{\mathbb{H}} \circ r_{\mathbb{H}}\right)\left(v_{\pi}\right)=\left(\mathrm{id} \otimes \tau_{-i}^{\mathbb{H}}\right)(w) \\
& =\left(F_{w}^{\mathbb{H}} \otimes 1\right) w\left(\left(F_{w}^{\mathbb{H}}\right)^{-1} \otimes 1\right) .
\end{aligned}
$$

This shows $k_{\pi}:=\left(F_{w}^{\mathbb{H}}\right)^{-1} F_{\pi}^{\mathbb{G}} \in B\left(H_{\pi}\right)$ is an intertwiner of $w$, and in particular, $F_{w}^{\mathbb{H}} k_{\pi}=k_{\pi} F_{w}^{\mathbb{H}}$ holds. Hence $F_{\pi}^{\mathbb{G}}=F_{w}^{\mathbb{H}} k_{\pi}=k_{\pi} F_{w}^{\mathbb{H}}$. Since the positive operators $F_{\pi}^{\mathbb{G}}$ and $\left(F_{w}^{\mathbb{H}}\right)^{-1}$ commute, $k_{\pi}$ is positive. In particular, we have $F_{\pi}^{i t}=\left(F_{w}^{\mathbb{H}}\right)^{i t} k_{\pi}^{i t}$ for all $t \in \mathbb{R}$. Hence for any $\pi \in \operatorname{Irr}(\mathbb{G})$, we have

$$
\begin{aligned}
\left(\mathrm{id} \otimes r_{\mathbb{H}} \circ \tau_{t}^{\mathbb{G}}\right)\left(v_{\pi}\right) & =\left(\mathrm{id} \otimes r_{\mathbb{H}}\right)\left(\left(\left(F_{\pi}^{\mathbb{G}}\right)^{i t} \otimes 1\right) v_{\pi}\left(\left(F_{\pi}^{\mathbb{G}}\right)^{-i t} \otimes 1\right)\right) \\
& =\left(\left(F_{\pi}^{\mathbb{G}}\right)^{i t} \otimes 1\right) w\left(\left(F_{\pi}^{\mathbb{G}}\right)^{-i t} \otimes 1\right) \\
& =\left(\left(F_{w}^{\mathbb{H}}\right)^{i t} k_{\pi}^{i t} \otimes 1\right) w\left(k_{\pi}^{-i t}\left(F_{w}^{\mathbb{H}}\right)^{-i t} \otimes 1\right) \\
& =\left(\left(F_{w}^{\mathbb{H}}\right)^{i t} \otimes 1\right) w\left(\left(F_{w}^{\mathbb{H}}\right)^{-i t} \otimes 1\right) \\
& =\left(\mathrm{id} \otimes \tau_{t}^{\mathbb{H}}\right)(w) \\
& =\left(\mathrm{id} \otimes \tau_{t}^{\mathbb{H}} \circ r_{\mathbb{H}}\right)\left(v_{\pi}\right) .
\end{aligned}
$$

Therefore the desired relation holds.

(3). It follows from $r_{\mathbb{H}} \circ \kappa_{\mathbb{G}}=\kappa_{\mathbb{H}} \circ r_{\mathbb{H}}$ and (2).

Let $\left\{\mathbb{H}, r_{\mathbb{H}}\right\}$ be an algebraic quantum subgroup of $\mathbb{G}$. Since $W_{\mathbb{G}}\left(1 \otimes 1_{\pi}\right) \in$ $A(\mathbb{G}) \otimes L(\mathbb{G}) 1_{\pi}$ for any $\pi \in \operatorname{Irr}(\mathbb{G})$, we can define a left unitary representation of $\mathbb{H},\left(r_{\mathbb{H}} \otimes \mathrm{id}\right)\left(W_{\mathbb{G}}\right) \in L^{\infty}(\mathbb{H}) \otimes L(\mathbb{G})$ by

$$
\left(r_{\mathbb{H}} \otimes \mathrm{id}\right)\left(W_{\mathbb{G}}\right)=\sum_{\pi \in \operatorname{Irr}(\mathbb{G})}\left(r_{\mathbb{H}} \otimes \mathrm{id}\right)\left(W_{\mathbb{G}}\left(1 \otimes 1_{\pi}\right)\right)
$$

Lemma 2.10. Let $\mathbb{G}$ and $\mathbb{H}$ be compact quantum groups.

(1) Assume that $\left\{\mathbb{H}, r_{\mathbb{H}}\right\}$ is an algebraic quantum subgroup of $\mathbb{G}$. Then there exists a faithful normal unital *-homomorphism $r_{\mathbb{H}}^{t}: L(\mathbb{H}) \rightarrow L(\mathbb{G})$ such that

$$
\left(\mathrm{id} \otimes r_{\mathbb{H}}^{t}\right)\left(W_{\mathbb{H}}\right)=\left(r_{\mathbb{H}} \otimes \mathrm{id}\right)\left(W_{\mathbb{G}}\right)
$$


(2) Assume that there exists a faithful normal unital *-homomorphism $\iota: L(\mathbb{H}) \rightarrow L(\mathbb{G})$ such that

$$
\Delta_{\mathbb{G}} \circ \iota=(\iota \otimes \iota) \circ \Delta_{\mathbb{H}} .
$$

Then there exists a restriction map $r_{\mathbb{H}}: A(\mathbb{G}) \rightarrow A(\mathbb{H})$ such that $\iota=r_{\mathbb{H}}^{t}$.

Proof. (1). Since the map $L^{\infty}(\mathbb{H})_{*} \ni \omega \mapsto(\omega \otimes \mathrm{id})\left(W_{\mathbb{H}}\right) \in L(\mathbb{H})$ is injective, we can define a linear map $r_{\mathbb{H}}^{t}: L(\mathbb{H})^{\mathrm{fin}} \rightarrow L(\mathbb{G})$ by

$$
r_{\mathbb{H}}^{t}\left((\omega \otimes \mathrm{id})\left(W_{\mathbb{H}}\right)\right)=(\omega \otimes \mathrm{id})\left(\left(r_{\mathbb{H}} \otimes \mathrm{id}\right)\left(W_{\mathbb{G}}\right)\right) \quad \text { for } \omega \in L^{\infty}(\mathbb{H})_{*}^{\mathrm{fin}} .
$$

It is easy to verify the multiplicativity of $r_{\mathbb{H}}^{t}$ by the pentagon equalities (2.3) on $W_{\mathbb{H}}$ and $W_{\mathbb{G}}$. We show that $r_{\mathbb{H}}^{t}$ preserves the involutions. Take any $\omega \in L^{\infty}(\mathbb{H})_{*}^{\text {fin }}$ and $\pi \in \operatorname{Irr}(\mathbb{G})$. Using the equality $r_{\mathbb{H}} \circ \kappa_{\mathbb{G}}=\kappa_{\mathbb{H}} \circ r_{\mathbb{H}}$, we have

$$
\begin{aligned}
r_{\mathbb{H}}^{t}\left((\omega \otimes \mathrm{id})\left(W_{\mathbb{H}}\right)\right)^{*}\left(1 \otimes 1_{\pi}\right) & =\left((\omega \otimes \mathrm{id})\left(\left(r_{\mathbb{H}} \otimes \mathrm{id}\right)\left(W_{\mathbb{G}}\right)\right)\right)^{*}\left(1 \otimes 1_{\pi}\right) \\
& =\left(\bar{\omega} \circ r_{\mathbb{H}} \otimes \mathrm{id}\right)\left(W_{\mathbb{G}}^{*}\left(1 \otimes 1_{\pi}\right)\right) \\
& =\left(\bar{\omega} \circ r_{\mathbb{H}} \otimes \mathrm{id}\right)\left(\left(\kappa_{\mathbb{G}} \otimes \mathrm{id}\right)\left(W_{\mathbb{G}}\right)\left(1 \otimes 1_{\pi}\right)\right) \\
& =\left(\bar{\omega} \circ \kappa_{\mathbb{H}} \otimes \mathrm{id}\right)\left(\left(r_{\mathbb{H}} \otimes \mathrm{id}\right)\left(W_{\mathbb{G}}\right)\left(1 \otimes 1_{\pi}\right)\right) \\
& =r_{\mathbb{H}}^{t}\left(\left(\bar{\omega} \circ \kappa_{\mathbb{H}} \otimes \mathrm{id}\right)\left(W_{\mathbb{H}}\right)\right)\left(1 \otimes 1_{\pi}\right) \\
& \left.=r_{\mathbb{H}}^{t}\left((\omega \otimes \mathrm{id})\left(W_{\mathbb{H}}\right)\right)^{*}\right)\left(1 \otimes 1_{\pi}\right) .
\end{aligned}
$$

Hence $r_{\mathbb{H}}^{t}$ is a $*$-homomorphism. Since $L(\mathbb{H})$ is a von Neumann algebra direct sum of $\left\{B\left(H_{\rho}\right)\right\}_{\rho \in \operatorname{Irr}(\mathbb{H})}$, the map $r_{\mathbb{H}}^{t}$ extends to a normal $*$-homomorphism from $L(\mathbb{H})$ to $L(\mathbb{G})$. Then the desired equality holds. We show that $r_{\mathbb{H}}^{t}$ is unital. Take any $\pi \in \operatorname{Irr}(\mathbb{G})$ and $\rho \in \operatorname{Irr}(\mathbb{H})$. Let $N_{\pi_{\mid \mathbb{H}}}^{\rho}$ be the multiplicity of $\rho$ in the unitary representation $\left(r_{\mathbb{H}} \otimes \mathrm{id}\right)\left(W_{\mathbb{G}}\left(1 \otimes 1_{\pi}\right)\right)$. Then the map $r_{\mathbb{H}}^{t}: L(\mathbb{H}) 1_{\rho} \rightarrow L(\mathbb{G}) 1_{\pi}$ is the $N_{\left.\pi\right|_{\mathbb{H}}}^{\rho}$ times amplification, and $r_{\mathbb{H}}^{t}$ is unital. Since $r_{\mathbb{H}}$ is surjective, $r_{\mathbb{H}}^{t}$ is injective.

(2). Since the linear map $L(\mathbb{G})_{*} \ni \omega \mapsto(\mathrm{id} \otimes \omega)\left(W_{\mathbb{G}}\right) \in L^{\infty}(\mathbb{G})$ is injective, we can define a linear map $r_{\mathbb{H}}: A(\mathbb{G}) \rightarrow A(\mathbb{H})$ by

$$
r_{\mathbb{H}}\left((\operatorname{id} \otimes \omega)\left(W_{\mathbb{G}}\right)\right)=(\operatorname{id} \otimes \omega \circ \iota)\left(W_{\mathbb{H}}\right) \quad \text { for } \omega \in L(\mathbb{G})_{*}^{\text {fin }} .
$$

First we show that $r_{\mathbb{H}}$ is multiplicative. Take any $\omega, \theta \in L(\mathbb{G})_{*}^{\text {fin }}$. Then $(\omega \otimes \theta) \circ$ $\Delta_{\mathbb{G}} \in L(\mathbb{G})_{*}^{\text {fin }}$, and

$$
\begin{aligned}
r_{\mathbb{H}}\left((\mathrm{id} \otimes \omega)\left(W_{\mathbb{G}}\right)\right) r_{\mathbb{H}}\left((\mathrm{id} \otimes \theta)\left(W_{\mathbb{G}}\right)\right) & =(\mathrm{id} \otimes \omega \circ \iota)\left(W_{\mathbb{H}}\right)(\mathrm{id} \otimes \theta \circ \iota)\left(W_{\mathbb{H}}\right) \\
& =(\mathrm{id} \otimes \omega \circ \iota \otimes \theta \circ \iota)\left(\left(W_{\mathbb{H}}\right)_{12}\left(W_{\mathbb{H}}\right)_{13}\right) \\
& =(\mathrm{id} \otimes \omega \circ \iota \otimes \theta \circ \iota)\left(\left(\mathrm{id} \otimes \Delta_{\mathbb{H}}\right)\left(W_{\mathbb{H}}\right)\right) \\
& =(\mathrm{id} \otimes \omega \otimes \theta)\left(\left(\mathrm{id} \otimes \Delta_{\mathbb{G}}\right)\left((\mathrm{id} \otimes \iota)\left(W_{\mathbb{H}}\right)\right)\right) \\
& =r_{\mathbb{H}}\left(\left(\mathrm{id} \otimes(\omega \otimes \theta) \circ \Delta_{\mathbb{G}}\right)\left(W_{\mathbb{G}}\right)\right) \\
& =r_{\mathbb{H}}\left((\mathrm{id} \otimes \omega)\left(W_{\mathbb{G}}\right)(\mathrm{id} \otimes \theta)\left(W_{\mathbb{G}}\right)\right) .
\end{aligned}
$$

Next we show $r_{\mathbb{H}}$ preserves the involutions. Let $\pi \in \operatorname{Irr}(\mathbb{G})$. Take $\omega \in L(\mathbb{G})_{*}^{\text {fin }}$ such that $\omega$ is equal to zero on $B\left(H_{\rho}\right)$ if $\rho \neq \bar{\pi}$. Since $\Delta_{\mathbb{G}} \circ \iota=(\iota \otimes \iota) \circ \Delta$, 
$(\mathrm{id} \otimes \iota)\left(W_{\mathbb{H}}\right)$ is a unitary representation of the discrete quantum group $(L(\mathbb{G}), \Delta)$. Set $\mathcal{V}=(\mathrm{id} \otimes \iota)\left(W_{\mathbb{H}}\right)$. By Lemma 2.4, we have $(\mathrm{id} \otimes \hat{S})\left(\mathcal{V}_{\pi}\right)=\mathcal{V}_{\bar{\pi}}^{*}$. Then

$$
\begin{aligned}
r_{\mathbb{H}}\left((\mathrm{id} \otimes \omega)\left(W_{\mathbb{G}}\right)\right)^{*} & =\left((\mathrm{id} \otimes \omega \circ \iota)\left(W_{\mathbb{H}}\right)\right)^{*}=(\mathrm{id} \otimes \bar{\omega} \circ \iota)\left(W_{\mathbb{H}}^{*}\right) \\
& =(\operatorname{id} \otimes \bar{\omega})\left(\mathcal{V}_{\bar{\pi}}^{*}\right)=(\mathrm{id} \otimes \bar{\omega} \circ \hat{S})\left(\mathcal{V}_{\pi}\right) \\
& =r_{\mathbb{H}}\left((\mathrm{id} \otimes \bar{\omega} \circ \hat{S})\left(\left(W_{\mathbb{G}}\right)_{\pi}\right)\right)=r_{\mathbb{H}}\left((\mathrm{id} \otimes \bar{\omega})\left(\left(W_{\mathbb{G}}\right)_{\bar{\pi}}^{*}\right)\right) \\
& =r_{\mathbb{H}}\left(\left((\mathrm{id} \otimes \omega)\left(W_{\mathbb{G}}\right)\right)^{*}\right) .
\end{aligned}
$$

By taking summations on $\omega$, we see that the above equality holds for any $\omega \in$ $L(\mathbb{G})_{*}^{\mathrm{fin}}$. Hence $r_{\mathbb{H}}$ is a unital $*$-homomorphism. Let $\mu \in L(\mathbb{H})_{*}^{\mathrm{fin}}$. Then there exists $\tilde{\mu} \in L(\mathbb{G})_{*}^{\text {fin }}$ such that $\mu=\tilde{\mu} \circ \iota$. Then we have $r_{\mathbb{H}}\left((\mathrm{id} \otimes \tilde{\mu})\left(W_{\mathbb{G}}\right)\right)=$ $(\mathrm{id} \otimes \mu)\left(W_{\mathbb{H}}\right)$. Hence $r_{\mathbb{H}}$ is surjective. Finally we show that $r_{\mathbb{H}}$ is a restriction map. Let $\omega \in L(\mathbb{G})_{*}^{\text {fin }}$. Then

$$
\begin{aligned}
\delta_{\mathbb{H}}\left(r_{\mathbb{H}}\left((\mathrm{id} \otimes \omega)\left(W_{\mathbb{G}}\right)\right)\right) & =\delta_{\mathbb{H}}\left((\mathrm{id} \otimes \omega \circ \iota)\left(W_{\mathbb{H}}\right)\right) \\
& =(\mathrm{id} \otimes \mathrm{id} \otimes \omega \circ \iota)\left(\left(W_{\mathbb{H}}\right)_{13}\left(W_{\mathbb{H}}\right)_{23}\right) \\
& =\left(r_{\mathbb{H}} \otimes r_{\mathbb{H}}\right)\left((\mathrm{id} \otimes \mathrm{id} \otimes \omega \circ \iota)\left(\left(W_{\mathbb{G}}\right)_{13}\left(W_{\mathbb{G}}\right)_{23}\right)\right) \\
& =\left(r_{\mathbb{H}} \otimes r_{\mathbb{H}}\right) \circ \delta_{\mathbb{G}}\left((\mathrm{id} \otimes \omega)\left(W_{\mathbb{H}}\right)\right) .
\end{aligned}
$$

Hence $\left\{\mathbb{H}, r_{\mathbb{H}}\right\}$ is an algebraic quantum subgroup of $\mathbb{G}$. It is clear that $\iota=r_{\mathbb{H}}^{t}$ by definition of $r_{\mathbb{H}}$.

On heredity of co-amenability to quantum subgroups, we have the following lemma.

Lemma 2.11. Let $\mathbb{G}$ be a compact quantum group. Then the following statements are equivalent:

(1) $\mathbb{G}$ is co-amenable.

(2) Any quantum subgroup of $\mathbb{G}$ is co-amenable.

(3) There exists a co-amenable quantum subgroup of $\mathbb{G}$.

Proof. The implication (2) to (3) is trivial. Suppose (3) holds. Let $\left\{\mathbb{H}, r_{\mathbb{H}}\right\}$ be a co-amenable quantum subgroup. Then $\varepsilon_{\mathbb{G}}=\varepsilon_{\mathbb{H}} \circ r_{\mathbb{H}}$ is bounded, and (1) holds. We have to show that (1) implies (2). Let $\left\{\mathbb{H}, r_{\mathbb{H}}\right\}$ be a quantum subgroup of $\mathbb{G}$. By the previous lemma, there exists an embedding map $r_{\mathbb{H}}^{t}: L(\mathbb{H}) \rightarrow L(\mathbb{G})$ preserving the coproducts. Let $m \in L(\mathbb{G})^{*}$ be a left invariant mean. Then the state $m \circ r_{\mathbb{H}}^{t} \in L(\mathbb{H})^{*}$ is also a left invariant mean on $L(\mathbb{H})$. Hence the discrete quantum group $(L(\mathbb{H}), \Delta)$ is amenable, and $\mathbb{H}$ is co-amenable.

Let $\left\{\mathbb{H}, r_{\mathbb{H}}\right\}$ be a quantum subgroup of $\mathbb{G}$. We define a map $\gamma_{\mathbb{H}}: C(\mathbb{G}) \rightarrow$ $C(\mathbb{H}) \otimes C(\mathbb{G})$ by $\gamma_{\mathbb{H}}(x)=\left(r_{\mathbb{H}} \otimes \mathrm{id}\right)\left(\delta_{\mathbb{G}}(x)\right)$ for $x \in C(\mathbb{G})$. Then $\gamma_{\mathbb{H}}$ is a left action of $\mathbb{H}$ on $C(\mathbb{G})$, that is, $\gamma_{\mathbb{H}}$ satisfies $\left(\mathrm{id} \otimes \gamma_{\mathbb{H}}\right) \circ \gamma_{\mathbb{H}}=\left(\delta_{\mathbb{H}} \otimes \mathrm{id}\right) \circ \gamma_{\mathbb{H}}$. We set the fixed point algebra $C(\mathbb{H} \backslash \mathbb{G})=\left\{x \in C(\mathbb{G}) \mid \gamma_{\mathbb{H}}(x)=1 \otimes x\right\}$. Since the action $\gamma_{\mathbb{H}}$ preserves the Haar state of $\mathbb{G}$, it extends to an action on $L^{\infty}(\mathbb{G})$. We denote the fixed point algebra by $L^{\infty}(\mathbb{H} \backslash \mathbb{G})$ as well. Then $L^{\infty}(\mathbb{H} \backslash \mathbb{G})$ is the range of the conditional expectation $E_{\mathbb{H}}=\left(h_{\mathbb{H}} \otimes \mathrm{id}\right) \circ \gamma_{\mathbb{H}}$. 
We often identify $L(\mathbb{H})$ with the subalgebra of $L(\mathbb{G})$ via the inclusion map $r_{\mathbb{H}}^{t}: L(\mathbb{H}) \rightarrow L(\mathbb{G})$ defined in Lemma 2.10, By the identification, we have $W_{\mathbb{H}}=$ $\left(r_{\mathbb{H}} \otimes \mathrm{id}\right)\left(W_{\mathbb{G}}\right)$. Similarly, we can do $R(\mathbb{H}) \subset R(\mathbb{G})$ and $\left(\mathrm{id} \otimes r_{\mathbb{H}}\right)\left(V_{\mathbb{G}}\right)=V_{\mathbb{H}}$. Then the left action of $\mathbb{H}$ on $L^{\infty}(\mathbb{G})$ is given by $\gamma_{\mathbb{H}}(x)=W_{\mathbb{H}}^{*}(1 \otimes x) W_{\mathbb{H}}$ for $x \in L^{\infty}(\mathbb{G})$.

Lemma 2.12. In the above setting, one has

$$
L(\mathbb{H})^{\prime} \cap L^{\infty}(\mathbb{G})=L^{\infty}(\mathbb{H} \backslash \mathbb{G}), \quad L^{\infty}(\mathbb{H} \backslash \mathbb{G})^{\prime} \cap L(\mathbb{G})=L(\mathbb{H}) .
$$

Proof. By definition of $r_{\mathbb{H}}, x \in L(\mathbb{H})^{\prime} \cap L^{\infty}(\mathbb{G})$ if and only if $W_{\mathbb{H}}^{*}(1 \otimes x) W_{\mathbb{H}}=1 \otimes x$. It is equivalent to $x \in L^{\infty}(\mathbb{H} \backslash \mathbb{G})$. Hence $L(\mathbb{H})^{\prime} \cap L^{\infty}(\mathbb{G})=L^{\infty}(\mathbb{H} \backslash \mathbb{G})$. By Lemma 2.6, we obtain the second equality.

Lemma 2.13. Let $\left\{\mathbb{H}, r_{\mathbb{H}}\right\}$ and $\left\{\mathbb{K}, r_{\mathbb{K}}\right\}$ be quantum subgroups of $\mathbb{G}$. Assume that $L^{\infty}(\mathbb{H} \backslash \mathbb{G})=L^{\infty}(\mathbb{K} \backslash \mathbb{G})$. Then there exists a *-isomorphism $\theta: C(\mathbb{H}) \rightarrow C(\mathbb{K})$ such that $r_{\mathbb{K}}=\theta \circ r_{\mathbb{H}}$ on $C(\mathbb{G})$.

Proof. By the previous lemma, we have $r_{\mathbb{H}}^{t}(L(\mathbb{H}))=r_{\mathbb{K}}^{t}(L(\mathbb{K}))$. Applying Lemma 2.10 to left group algebra $r_{\mathbb{H}}^{t}(L(\mathbb{H}))$, we have a $*$-isomorphism $\theta: C(\mathbb{H}) \rightarrow C(\mathbb{K})$ such that $(\theta \otimes \mathrm{id})\left(\left(\mathrm{id} \otimes r_{\mathbb{H}}^{t}\right)\left(W_{\mathbb{H}}\right)\right)=\left(\mathrm{id} \otimes r_{\mathbb{K}}^{t}\right)\left(W_{\mathbb{K}}\right)$. This implies $\left(\theta \circ r_{\mathbb{H}} \otimes \mathrm{id}\right)\left(W_{\mathbb{G}}\right)=$ $\left(r_{\mathbb{K}} \otimes \mathrm{id}\right)\left(W_{\mathbb{K}}\right)$, and $r_{\mathbb{K}}=\theta \circ r_{\mathbb{H}}$ on $C(\mathbb{G})$.

\section{Right COIDEALS OF QUOTIEnT TYPE}

Definition 3.1. Let $B \subset L^{\infty}(\mathbb{G})$ be a right coideal. We say that

(1) $B$ is of quotient type if there exists a quantum subgroup $\left\{\mathbb{H}, r_{\mathbb{H}}\right\}$ of $\mathbb{G}$ such that $B=L^{\infty}(\mathbb{H} \backslash \mathbb{G})$,

(2) $B$ has the expectation property if there exists a faithful normal conditional expectation $E_{B}: L^{\infty}(\mathbb{G}) \rightarrow B$ such that $h \circ E_{B}=h$,

(3) $B$ has the coaction symmetry if $\beta(B) \subset R(\mathbb{G}) \otimes B$.

Typical examples of right coideals are given by taking quotients. In fact, they have the expectation property and the coaction symmetry as follows.

Lemma 3.2. A right coideal of quotient type has the expectation property and the coaction symmetry.

Proof. Let $\left\{\mathbb{H}, r_{\mathbb{H}}\right\}$ be a quantum subgroup of $\mathbb{G}$. Set $B=L^{\infty}(\mathbb{H} \backslash \mathbb{G})$. It is easy to see that the conditional expectation $E_{\mathbb{H}}$ preserves the Haar state $h$. Next we verify the coaction symmetry. Let $x \in C(\mathbb{H} \backslash \mathbb{G})$. Then we have

$$
\begin{aligned}
\left(\mathrm{id} \otimes \gamma_{\mathbb{H}}\right)(\beta(x)) & =\left(\mathrm{id} \otimes\left(r_{\mathbb{H}} \otimes \mathrm{id}\right) \circ \delta\right)(\beta(x)) \\
& =\left(\mathrm{id} \otimes\left(r_{\mathbb{H}} \otimes \mathrm{id}\right) \circ \delta\right)\left(V_{\mathbb{G}}^{*}(1 \otimes x) V_{\mathbb{G}}\right) \\
& =\left(\mathrm{id} \otimes r_{\mathbb{H}} \otimes \mathrm{id}\right)\left(\left(V_{\mathbb{G}}\right)_{13}^{*}\left(V_{\mathbb{G}}\right)_{12}^{*}(1 \otimes \delta(x))\left(V_{\mathbb{G}}\right)_{12}\left(V_{\mathbb{G}}\right)_{13}\right) \\
& =\left(V_{\mathbb{G}}\right)_{13}^{*}\left(V_{\mathbb{H}}\right)_{12}^{*}(1 \otimes 1 \otimes x)\left(V_{\mathbb{H}}\right)_{12}\left(V_{\mathbb{G}}\right)_{13} \\
& =\left(V_{\mathbb{G}}\right)_{13}^{*}(1 \otimes 1 \otimes x)\left(V_{\mathbb{G}}\right)_{13} \\
& =\beta(x)_{13} .
\end{aligned}
$$


Hence $\beta(x) \in R(\mathbb{G}) \otimes L^{\infty}(\mathbb{H} \backslash \mathbb{G})$. By weak continuity of $\beta, \beta\left(L^{\infty}(\mathbb{H} \backslash \mathbb{G})\right) \subset$ $R(\mathbb{G}) \otimes L^{\infty}(\mathbb{H} \backslash \mathbb{G})$.

In fact assuming the co-amenability $\mathbb{G}$, We prove the converse statement of the previous lemma in Theorem 3.18.

Let $B \subset L^{\infty}(\mathbb{G})$ be a right coideal. We denote by $L^{2}(B)$ the norm closure of the space $B \hat{1}_{h}$. Assume that $B$ has the expectation property, that is, there exists a conditional expectation $E_{B}: L^{\infty}(\mathbb{G}) \rightarrow B$ such that $E_{B}$ preserves the Haar state $h$. Define the Jones projection $e_{B}: L^{2}(\mathbb{G}) \rightarrow L^{2}(B)$ by

$$
e_{B}\left(x \hat{1}_{h}\right)=E_{B}(x) \hat{1}_{h} \quad \text { for } x \in L^{\infty}(\mathbb{G}) .
$$

Let $B \subset L^{\infty}(\mathbb{G}) \subset L^{\infty}(\mathbb{G}) \vee\left\{e_{B}\right\}^{\prime \prime}=: B_{1}$ be the basic extension. The main properties of $e_{B}$ are as follows (see [13, Lemma 3.2] and [21, p. 312]).

Lemma 3.3. With the above settings, one has

(1) $e_{B} x e_{B}=E_{B}(x) e_{B}$ for $x \in L^{\infty}(\mathbb{G})$,

(2) $B=L^{\infty}(\mathbb{G}) \cap\left\{e_{B}\right\}^{\prime}$,

(3) $J e_{B}=e_{B} J, \Delta_{h}^{i t} e_{B}=e_{B} \Delta_{h}^{i t}$ for all $t \in \mathbb{R}$,

(4) $B_{1}=J B^{\prime} J$

Set $\widehat{B}=B^{\prime} \cap L(\mathbb{G})$. Then $\widehat{B}$ is a left coideal of $L(\mathbb{G})$ as is shown in Lemma [2.6. The following lemma is proved in [11, Theorem 4.6], which treats the Kac algebra case. The proof can be adapted to the quantum group case.

Lemma 3.4. With the above setting, one has

(1) $e_{B} \in \widehat{B}$,

(2) $\delta\left(E_{B}(x)\right)=\left(E_{B} \otimes \mathrm{id}\right)(\delta(x))$ for all $x \in L^{\infty}(\mathbb{G})$,

(3) $\widehat{B}^{\prime} \cap L^{\infty}(\mathbb{G})=B$.

Proof. (1). Since $B$ is a right coideal, we see that $V_{\mathbb{G}}\left(L^{2}(B) \otimes L^{2}(\mathbb{G})\right) \subset L^{2}(B) \otimes$ $L^{2}(\mathbb{G})$. Hence $V_{\mathbb{G}}\left(e_{B} \otimes 1\right)=\left(e_{B} \otimes 1\right) V_{\mathbb{G}}\left(e_{B} \otimes 1\right)$. Since $(J \otimes \hat{J}) V_{\mathbb{G}}(J \otimes \hat{J})=V_{\mathbb{G}}^{*}$ and $J e_{B} J=e_{B}, V_{\mathbb{G}}\left(e_{B} \otimes 1\right)=\left(e_{B} \otimes 1\right) V_{\mathbb{G}}$. Hence $e_{B} \in B^{\prime} \cap L(\mathbb{G})=\widehat{B}$.

(2). Let $x \in L^{\infty}(\mathbb{G})$. Since $e_{B} \in L(\mathbb{G})$ and $V_{\mathbb{G}} \in R(\mathbb{G}) \otimes L^{\infty}(\mathbb{G})$, we have

$$
\begin{aligned}
\left(E_{B} \otimes \mathrm{id}\right)(\delta(x))\left(e_{B} \otimes 1\right) & =\left(e_{B} \otimes 1\right) \delta(x)\left(e_{B} \otimes 1\right)=\left(e_{B} \otimes 1\right) V_{\mathbb{G}}(x \otimes 1) V_{\mathbb{G}}^{*}\left(e_{B} \otimes 1\right) \\
& =V_{\mathbb{G}}\left(e_{B} \otimes 1\right)(x \otimes 1)\left(e_{B} \otimes 1\right) V_{\mathbb{G}}^{*}=V_{\mathbb{G}}\left(E_{B}(x) e_{B} \otimes 1\right) V_{\mathbb{G}}^{*} \\
& =V_{\mathbb{G}}\left(E_{B}(x) \otimes 1\right) V_{\mathbb{G}}^{*}\left(e_{B} \otimes 1\right) \\
& =\delta\left(E_{B}(x)\right)\left(e_{B} \otimes 1\right) .
\end{aligned}
$$

In particular, we have $\delta\left(E_{B}(x)\right)\left(\hat{1}_{h} \otimes \hat{1}_{h}\right)=\left(E_{B} \otimes \mathrm{id}\right)(\delta(x))\left(\hat{1}_{h} \otimes \hat{1}_{h}\right)$. Since $\hat{1}_{h} \otimes \hat{1}_{h}$ is a separating vector for $L^{\infty}(\mathbb{G}) \otimes L^{\infty}(\mathbb{G})$, we have $\delta\left(E_{B}(x)\right)=\left(E_{B} \otimes \mathrm{id}\right)(\delta(x))$.

(3). It follows from Lemma 2.6 or the direct argument as follows. It is clear that $B \subset \widehat{B}^{\prime} \cap L^{\infty}(\mathbb{G})$. Since $e_{B} \in \widehat{B}$ and $B=\left\{e_{B}\right\}^{\prime} \cap L^{\infty}(\mathbb{G}), B=\widehat{B}^{\prime} \cap L^{\infty}(\mathbb{G})$.

Next we consider a relation between $\widehat{B}$ and $B_{1}$. Let $\alpha$ be the right $\mathbb{G}$-action $\alpha$ on $B\left(L^{2}(\mathbb{G})\right.$ ) defined in $\$ 2.4$. Since $e_{B} \otimes 1$ commutes with $V_{\mathbb{G}}, B_{1}$ is globally 
invariant under the right action $\alpha$. Let $X$ be a globally invariant subspace in $B_{1}$. The set of the fixed point elements of $X$ under $\alpha$ is denoted by $X^{\mathbb{G}}$. Note that $X^{\mathbb{G}}=X \cap L(\mathbb{G})$ holds.

Lemma 3.5. The following equalities hold:

(1) $\hat{R}\left(e_{B}\right)=e_{B}$.

(2) $\left(e_{B} \otimes 1\right) W_{\mathbb{G}}^{*}\left(1 \otimes e_{B}\right)=W_{\mathbb{G}}^{*}\left(e_{B} \otimes e_{B}\right)$.

(3) $\widehat{B}=J B_{1}^{\mathbb{G}} J=\overline{\mathbb{C}+J\left(L^{\infty}(\mathbb{G}) e_{B} L^{\infty}(\mathbb{G})\right)^{\mathbb{G}}{ }^{\mathrm{w}}}$.

Proof. (1). Since $e_{B}^{*}=e_{B}$ and $J e_{B}=e_{B} J$, we have $\hat{R}\left(e_{B}\right)=J e_{B}^{*} J=e_{B}$.

(2). Take any $x, y \in L^{\infty}(\mathbb{G})$. Then we have

$$
\begin{aligned}
\left(e_{B} \otimes 1\right) W_{\mathbb{G}}^{*}\left(1 \otimes e_{B}\right)\left(x \hat{1}_{h} \otimes y \hat{1}_{h}\right) & =\left(e_{B} \otimes 1\right) W_{\mathbb{G}}^{*}\left(x \hat{1}_{h} \otimes E_{B}(y) \hat{1}_{h}\right) \\
& =\left(e_{B} \otimes 1\right)\left(\delta\left(E_{B}(y)\right) x \hat{1}_{h} \otimes \hat{1}_{h}\right) \\
& =\delta\left(E_{B}(y)\right)\left(E_{B}(x) \hat{1}_{h} \otimes \hat{1}_{h}\right) \\
& =W_{\mathbb{G}}^{*}\left(e_{B} \otimes e_{B}\right)\left(x \hat{1}_{h} \otimes y \hat{1}_{h}\right) .
\end{aligned}
$$

Hence the desired equality holds.

(3). Since $B_{1}=J B^{\prime} J$ and $J L(\mathbb{G}) J=L(\mathbb{G})$, we have

$$
\begin{aligned}
J \widehat{B} J & =J\left(B^{\prime} \cap L(\mathbb{G})\right) J=J B^{\prime} J \cap J L(\mathbb{G}) J \\
& =B_{1} \cap L(\mathbb{G})=B_{1}^{\mathbb{G}} .
\end{aligned}
$$

By Lemma 3.4, the $*$-subalgebra $L^{\infty}(\mathbb{G})+L^{\infty}(\mathbb{G}) e_{B} L^{\infty}(\mathbb{G})$ is weakly dense in $B_{1}$. Hence we have $B_{1}^{\mathbb{G}}=\overline{\mathbb{C}+\left(L^{\infty}(\mathbb{G}) e_{B} L^{\infty}(\mathbb{G})\right)^{\mathbb{G}}}$.

Lemma 3.6. One has

$$
\left(A(\mathbb{G}) e_{B} A(\mathbb{G})\right)^{\mathbb{G}}=\left\{(\operatorname{id} \otimes \omega)\left(\Delta\left(e_{B}\right)\right) \mid \omega \in L(\mathbb{G})_{*}^{\text {fin }}\right\} .
$$

Proof. Let $\pi, \sigma \in \operatorname{Irr}(\mathbb{G}), i, j \in I_{\pi}$ and $k, \ell \in I_{\rho}$. Then

$$
\begin{aligned}
E_{\alpha}\left(v_{\pi_{i, j}} e_{B} v_{\rho_{k, \ell}}^{*}\right) & =(\operatorname{id} \otimes h)\left(\alpha\left(v_{\pi_{i, j}} e_{B} v_{\rho_{k, \ell}}^{*}\right)\right) \\
& =\sum_{m \in I_{\pi}} \sum_{n \in I_{\rho}} v_{\pi_{i, m}} e_{B} v_{\rho_{k, n}}^{*} h\left(v_{\pi_{m, j}} v_{\rho_{n, \ell}}^{*}\right) \\
& =\sum_{m \in I_{\pi}} \sum_{n \in I_{\rho}} \delta_{\pi, \rho} \delta_{m, n} \delta_{j, \ell} D_{\pi}^{-1} F_{\pi_{j, j}} v_{\pi_{i, m}} e_{B} v_{\rho_{k, n}}^{*} \\
& =D_{\pi}^{-1} F_{\pi_{j, j}} \delta_{\pi, \rho} \delta_{j, \ell} \sum_{m \in I_{\pi}} v_{\pi_{i, m}} e_{B} v_{\pi_{k, m}}^{*} .
\end{aligned}
$$

Hence

$$
\left(A(\mathbb{G}) e_{B} A(\mathbb{G})\right)^{\mathbb{G}}=\operatorname{span}\left\{\sum_{m \in I_{\pi}} v_{\pi_{i, m}} e_{B} v_{\pi_{k, m}}^{*} \mid i, k \in I_{\pi}, \pi \in \operatorname{Irr}(\mathbb{G})\right\} .
$$

Using the description of $W_{\mathbb{G}}$ in (2.10), we have

$$
\Delta\left(e_{B}\right)\left(1 \otimes 1_{\pi}\right)=\sum_{i, j, k \in I_{\pi}} v_{\pi_{i, j}} e_{B} v_{\pi_{k, j}}^{*} \otimes f_{\pi_{i, k}} .
$$


Hence

$$
\left(A(\mathbb{G}) e_{B} A(\mathbb{G})\right)^{\mathbb{G}}=\left\{(\operatorname{id} \otimes \omega)\left(\Delta\left(e_{B}\right)\right) \mid \omega \in L(\mathbb{G})_{*}^{\text {fin }}\right\} .
$$

Lemma 3.7. One has $B_{1}^{\mathbb{G}}={\overline{\left(L^{\infty}(\mathbb{G}) e_{B} L^{\infty}(\mathbb{G})\right)^{\mathbb{G}}}}^{\mathrm{w}}$.

Proof. By Lemma 3.5, it suffices to show that the unit of $B_{1}$ is equal to that of $\overline{\left(L^{\infty}(\mathbb{G}) e_{B} L^{\infty}(\mathbb{G})\right)^{\mathbb{G}^{\mathrm{w}}}}$. Let $p \in B_{1}^{\mathbb{G}}$ be the unit of the von Neumann subalgebra generated by $\left(L^{\infty}(\mathbb{G}) e_{B} L^{\infty}(\mathbb{G})\right)^{\mathbb{G}}$. Set $q=1-p$. Then by the previous lemma, we have $\Delta\left(e_{B}\right)(q \otimes 1)=0$. Since $\hat{R}\left(e_{B}\right)=e_{B}$ by Lemma 3.5, we have

$$
\begin{aligned}
(1 \otimes \hat{R}(q)) \Delta\left(e_{B}\right) & =(1 \otimes \hat{R}(q)) \Delta\left(\hat{R}\left(e_{B}\right)\right)=(1 \otimes \hat{R}(q))(\hat{R} \otimes \hat{R})\left(\Delta^{\mathrm{op}}\left(e_{B}\right)\right) \\
& =(\hat{R} \otimes \hat{R})\left(\Delta^{\mathrm{op}}\left(e_{B}\right)(1 \otimes q)\right)=0 .
\end{aligned}
$$

The equality $\Delta\left(e_{B}\right)=W_{\mathbb{G}}\left(e_{B} \otimes 1\right) W_{\mathbb{G}}^{*}$ yields $(1 \otimes \hat{R}(q)) W_{\mathbb{G}}\left(e_{B} \otimes 1\right)=0$, and $\left(E_{B} \otimes \mathrm{id}\right)\left(W_{\mathbb{G}}^{*}(1 \otimes \hat{R}(q)) W_{\mathbb{G}}\right)=0$. Since $E_{B}$ is faithful, we have $q=0$.

Lemma 3.8. One has

$$
B_{1}^{\mathbb{G}}={\overline{\left\{(\mathrm{id} \otimes \omega)\left(\Delta\left(e_{B}\right)\right) \mid \omega \in L(\mathbb{G})_{*}\right.}}^{\mathrm{w}}, \quad \widehat{B}={\overline{\left\{(\omega \otimes \mathrm{id})\left(\Delta\left(e_{B}\right)\right) \mid \omega \in L(\mathbb{G})_{*}\right.}}^{\mathrm{w}} .
$$

Proof. The left equality follows from Lemma 3.6 and the previous lemma. Take $\omega \in L(\mathbb{G})_{*}$. By (2.17) and Lemma 3.5, we have

$$
J(\mathrm{id} \otimes \omega)\left(\Delta\left(e_{B}\right)\right) J=(\mathrm{id} \otimes \bar{\omega} \circ \hat{R})\left((\hat{R} \otimes \hat{R})\left(\Delta\left(e_{B}\right)\right)\right)=(\bar{\omega} \circ \hat{R} \otimes \mathrm{id})\left(\Delta\left(e_{B}\right)\right)
$$

Hence the right equality holds since $\widehat{B}=J B_{1}^{\mathbb{G}} J$.

To construct a left invariant weight on $\widehat{B}$, we make use of theory of spatial derivatives and operator valued weights which have been introduced in [5], 8] and [9]. Readable explanations on them are presented in [14] and we freely use the notations there. Let $E_{B}^{-1}: B^{\prime} \rightarrow L^{\infty}(\mathbb{G})^{\prime}$ be the operator valued weights associated with $E_{B}: L^{\infty}(\mathbb{G}) \rightarrow B$. It is characterized by the following equality on spatial derivatives:

$$
\frac{d \omega^{\prime} \circ E_{B}^{-1}}{d \omega}=\frac{d \omega^{\prime}}{d \omega \circ E_{B}}
$$

where $\omega^{\prime}$ and $\omega$ are faithful normal semifinite weights on $L^{\infty}(\mathbb{G})^{\prime}$ and $B$, respectively. The equality $E_{B}^{-1}\left(e_{B}\right)=1$ holds [13, Lemma 3.1]. We define the *-subalgebra $\widehat{B}_{0}$ of $\widehat{B}$ by

$$
\widehat{B}_{0}=J\left(A(\mathbb{G}) e_{B} A(\mathbb{G})\right)^{\mathbb{G}} J=\left\{(\omega \otimes \mathrm{id})\left(\Delta\left(e_{B}\right)\right) \mid \omega \in L(\mathbb{G})_{*}^{\mathrm{fin}}\right\} .
$$

Lemma 3.9. The operator valued weight $E_{B}^{-1}$ is finite on $\widehat{B}_{0}$. In particular, $E_{B}^{-1}$ is semifinite on $\widehat{B}$. 
Proof. Set a unitary $W_{\widehat{\mathbb{G}}}=(J \otimes J)\left(W_{\mathbb{G}}\right)_{21}^{*}(J \otimes J) \in L(\mathbb{G}) \otimes L^{\infty}(\mathbb{G})^{\prime}$. Then for any $x \in L(\mathbb{G})$, we have

$$
\begin{aligned}
W_{\overparen{\mathbb{G}}}^{*}(1 \otimes x) W_{\widehat{\mathbb{G}}} & =(J \otimes J)\left(W_{\mathbb{G}}\right)_{21}(1 \otimes J x J)\left(W_{\mathbb{G}}\right)_{21}^{*}(J \otimes J) \\
& =(J \otimes J)\left(W_{\mathbb{G}}\right)_{21}\left(1 \otimes \hat{R}\left(x^{*}\right)\right)\left(W_{\mathbb{G}}\right)_{21}^{*}(J \otimes J) \\
& =(J \otimes J) \Delta\left(\hat{R}\left(x^{*}\right)\right)_{21}(J \otimes J) \\
& =(\hat{R} \otimes \hat{R})\left(\Delta(\hat{R}(x))_{21}\right) \\
& =\Delta(x) .
\end{aligned}
$$

Now take a positive functional $\omega \in L(\mathbb{G})_{*}^{\text {fin }}$. Then by $L^{\infty}(\mathbb{G})^{\prime}$-linearity of $E_{B}^{-1}$, we have

$$
\begin{aligned}
E_{B}^{-1}\left((\omega \otimes \mathrm{id})\left(\Delta\left(e_{B}\right)\right)\right) & =E_{B}^{-1}\left((\omega \otimes \mathrm{id})\left(W_{\overparen{\mathbb{G}}}^{*}\left(1 \otimes e_{B}\right) W_{\widehat{\mathbb{G}}}\right)\right) \\
& =(\omega \otimes \mathrm{id})\left(W_{\overparen{\mathbb{G}}}^{*}\left(1 \otimes E_{B}^{-1}\left(e_{B}\right)\right) W_{\widehat{\mathbb{G}}}\right) \\
& =\omega(1)<\infty .
\end{aligned}
$$

Hence $E_{B}^{-1}$ is finite on $\widehat{B}_{0}$.

Define a state $h^{\prime} \in L^{\infty}(\mathbb{G})^{\prime}$ by $h^{\prime}(x)=h\left(J x^{*} J\right)$ for $x \in L^{\infty}(\mathbb{G})^{\prime}$. Set a faithful normal semifinite weight $\varphi=h^{\prime} \circ E_{B}^{-1}$ on $B^{\prime}$. We denote by $\varphi_{\widehat{B}}$ the restriction $\varphi$ on $\widehat{B}$.

Lemma 3.10. The modular automorphism group $\left\{\sigma_{t}^{\varphi_{\widehat{B}}}\right\}_{t \in \mathbb{R}}$ preserves $\widehat{B}_{0}$.

Proof. By (3.1), we obtain

$$
\frac{d \varphi}{d h_{B}}=\frac{d h^{\prime} \circ E_{B}^{-1}}{\left.d h\right|_{B}}=\frac{d h^{\prime}}{\left.d h\right|_{B} \circ E_{B}}=\frac{d h^{\prime}}{d h}=\Delta_{h}^{-1} .
$$

Hence $\sigma_{t}^{\varphi}=\operatorname{Ad} \Delta_{h}^{-i t}$ on $B^{\prime}$. Since $\operatorname{Ad} \Delta_{h}^{-i t}=\hat{\tau}_{t}$ on $L(\mathbb{G})$ by (2.15) and (2.16), the von Neumann subalgebra $\widehat{B} \subset B^{\prime}$ is globally invariant under $\sigma_{t}^{\varphi}$. Hence $\sigma_{t}^{\varphi_{\widehat{B}}}=\left.\sigma_{t}^{\varphi}\right|_{\widehat{B}}=\left.\hat{\tau}_{t}\right|_{\widehat{B}}$ by uniqueness of the modular automorphism group. Since $e_{B}$ commutes with $\Delta_{h}^{-i t}, \hat{\tau}_{t}\left(e_{B}\right)=\Delta_{h}^{-i t} e_{B} \Delta_{h}^{i t}=e_{B}$. Then for any $\omega \in L(\mathbb{G})_{*}^{\text {fin }}$,

$$
\sigma_{t}^{\varphi_{\widehat{B}}}\left((\omega \otimes \mathrm{id})\left(\Delta\left(e_{B}\right)\right)\right)=\hat{\tau}_{t}\left((\omega \otimes \mathrm{id})\left(\Delta\left(e_{B}\right)\right)\right)=\left(\omega \circ \hat{\tau}_{-t} \otimes \mathrm{id}\right)\left(\Delta\left(e_{B}\right)\right)
$$

by (2.17) and $\hat{\tau}_{t}\left(e_{B}\right)=e_{B}$. By definition of $\widehat{B}_{0}(\underline{3.2})$, we have $\sigma_{t}^{\varphi_{\widehat{B}}}\left(\widehat{B}_{0}\right) \subset \widehat{B}_{0}$.

By previous lemma, the following push-down lemma is proved in a similar way to [11, Proposition 2.2].

Lemma 3.11. For any $x \in n_{\varphi_{\widehat{B}}}, e_{B} x=e_{B} E_{B}^{-1}\left(e_{B} x\right)$ holds.

Lemma 3.12. One has $\left(A(\mathbb{G}) e_{B} L^{\infty}(\mathbb{G})\right)^{\mathbb{G}}=\left(A(\mathbb{G}) e_{B} A(\mathbb{G})\right)^{\mathbb{G}}$.

Proof. It suffices to show $\left(L^{\infty}(\mathbb{G})_{\pi} e_{B} L^{\infty}(\mathbb{G})\right)^{\mathbb{G}} \subset L^{\infty}(\mathbb{G})_{\pi} e_{B} L^{\infty}(\mathbb{G})_{\pi}^{*}$, where $L^{\infty}(\mathbb{G})_{\pi}$ is the linear span of $\left\{v_{\pi_{i, j}}\right\}_{i, j \in I_{\pi}}$. Take any $x \in L^{\infty}(\mathbb{G})$. Then

$$
E_{\alpha}\left(v_{\pi_{i, j}} e_{B} x\right)=\sum_{k \in I_{\pi}} v_{\pi_{i, k}} e_{B}(\mathrm{id} \otimes h)\left(\left(1 \otimes v_{\pi_{k, j}}\right) \delta(x)\right) \in L^{\infty}(\mathbb{G})_{\pi} e_{B} L^{\infty}(\mathbb{G})_{\pi}^{*},
$$


because $(\mathrm{id} \otimes h)\left(\left(1 \otimes v_{\pi_{k, j}}\right) \delta(x)\right) \in L^{\infty}(\mathbb{G})_{\pi}^{*}$ for any $x \in L^{\infty}(\mathbb{G})$.

Lemma 3.13. The weight $\varphi_{\widehat{B}}$ is left invariant, that is, for any $x \in m_{\varphi_{\widehat{B}}}$ and $\omega \in L(\mathbb{G})_{*}$, one has

$$
\left(\omega \otimes \varphi_{\widehat{B}}\right)(\Delta(x))=\omega(1) \varphi_{\widehat{B}}(x) .
$$

Proof. Take $\theta \in L(\mathbb{G})_{*}^{\text {fin }}$ and set $x=(\theta \otimes \mathrm{id})\left(\Delta\left(e_{B}\right)\right) \in \widehat{B}_{0}$. By using the proof of Lemma 3.9, we have $E_{B}^{-1}(x)=\theta(1)$. Then for any $\omega \in L(\mathbb{G})_{*}$,

$$
\begin{aligned}
\left(\omega \otimes \varphi_{\widehat{B}}\right)(\Delta(x)) & =\left(\omega \otimes h^{\prime} \circ E_{B}^{-1}\right)\left(W_{\widehat{\mathbb{G}}}^{*}(1 \otimes x) W_{\widehat{\mathbb{G}}}\right) \\
& =\left(\omega \otimes h^{\prime}\right)\left(W_{\overparen{\mathbb{G}}}^{*}\left(1 \otimes E_{B}^{-1}(x)\right) W_{\widehat{\mathbb{G}}}\right) \\
& =\omega(1) \theta(1) \\
& =\omega(1) \varphi_{\widehat{B}}(x) .
\end{aligned}
$$

Hence on $\widehat{B}_{0}$, the left invariance holds.

Take a bounded sequence $\left\{u_{n}\right\}_{n \in \mathbb{N}}$ in $\widehat{B}_{0}$ which strongly converges to 1 . Let $y \in n_{\varphi_{\widehat{B}}}$. Then by Lemma 3.11, $u_{n} y \in J\left(A(\mathbb{G}) e_{B} L^{\infty}(\mathbb{G})\right) J$ for each $n \in \mathbb{N}$. Since $J u_{n} y J \in J \widehat{B} J=B_{1}^{\mathbb{G}}$, we see that $u_{n} y \in J\left(A(\mathbb{G}) e_{B} L^{\infty}(\mathbb{G})\right)^{\mathbb{G}} J$. Using Lemma 3.12, we see that $u_{n} y \in J\left(A(\mathbb{G}) e_{B} A(\mathbb{G})\right)^{\mathbb{G}} J=\widehat{B}_{0}$. Hence for any positive $\omega \in L(\mathbb{G})_{*}$, we have

$$
\left(\omega \otimes \varphi_{\widehat{B}}\right)\left(\Delta\left(y^{*} u_{n}^{*} u_{n} y\right)\right)=\omega(1) \varphi_{\widehat{B}}\left(y^{*} u_{n}^{*} u_{n} y\right) .
$$

Taking the limit as $n \rightarrow \infty$ in (3.3), we have

$$
\begin{aligned}
\left(\omega \otimes \varphi_{\widehat{B}}\right)\left(\Delta\left(y^{*} y\right)\right) & \leq \lim _{n \rightarrow \infty}\left(\omega \otimes \varphi_{\widehat{B}}\right)\left(\Delta\left(y^{*} u_{n}^{*} u_{n} y\right)\right) \\
& =\lim _{n \rightarrow \infty} \omega(1) \varphi_{\widehat{B}}\left(y^{*} u_{n}^{*} u_{n} y\right) \\
& =\omega(1) \varphi_{\widehat{B}}\left(y^{*} y\right)<\infty .
\end{aligned}
$$

In particular, the map $x \in \widehat{B} \mapsto\left(\omega \otimes \varphi_{\widehat{B}}\right)\left(\Delta\left(y^{*} x y\right)\right)$ is a normal functional. Hence again taking the limit as $n \rightarrow \infty$ in (3.3), we have

$$
\left(\omega \otimes \varphi_{\widehat{B}}\right)\left(\Delta\left(y^{*} y\right)\right)=\omega(1) \varphi_{\widehat{B}}\left(y^{*} y\right) .
$$

Therefore $\varphi_{\widehat{B}}$ is left invariant on $m_{\varphi_{\widehat{B}}}$.

We summarize our arguments as follows.

Theorem 3.14. Let $B \subset L^{\infty}(\mathbb{G})$ be a right coideal. If $B$ has the expectation property, then the left coideal $\widehat{B}$ has a left invariant faithful normal semifinite weight for the left action of $L(\mathbb{G})$.

Next we study a right coideal of $\mathbb{G}$ endowed with the coaction symmetry.

Lemma 3.15. Let $B \subset L^{\infty}(\mathbb{G})$ be a right coideal. Then $B$ has the coaction symmetry if and only if $\Delta(\widehat{B}) \subset \widehat{B} \otimes \widehat{B}$. 
Proof. We know $\Delta(\widehat{B}) \subset L(\mathbb{G}) \otimes \widehat{B}$. Let $x \in B$ and $y \in \widehat{B}$. Then we have

$$
\begin{aligned}
\Delta(y)(x \otimes 1) & =W_{\mathbb{G}}(y \otimes 1) W_{\mathbb{G}}^{*}(x \otimes 1) \\
& =\left(1 \otimes U_{\mathbb{G}}\right)\left(V_{\mathbb{G}}\right)_{21}\left(1 \otimes U_{\mathbb{G}}\right)(y \otimes 1)\left(1 \otimes U_{\mathbb{G}}\right)\left(V_{\mathbb{G}}\right)_{21}^{*}\left(1 \otimes U_{\mathbb{G}}\right)(x \otimes 1) \\
& =\left(1 \otimes U_{\mathbb{G}}\right)\left(V_{\mathbb{G}}\right)_{21}(y \otimes 1) \beta(x)_{21}\left(V_{\mathbb{G}}\right)_{21}^{*}\left(1 \otimes U_{\mathbb{G}}\right)
\end{aligned}
$$

and similarly

$$
(x \otimes 1) \Delta(y)=\left(1 \otimes U_{\mathbb{G}}\right)\left(V_{\mathbb{G}}\right)_{21} \beta(x)_{21}(y \otimes 1)\left(V_{\mathbb{G}}\right)_{21}^{*}\left(1 \otimes U_{\mathbb{G}}\right) .
$$

Hence $\Delta(\widehat{B}) \subset \widehat{B} \otimes \widehat{B}$ if and only if $\beta(B) \subset R(\mathbb{G}) \otimes\left(\widehat{B}^{\prime} \cap L^{\infty}(\mathbb{G})\right)=R(\mathbb{G}) \otimes B$.

Set a weight $\psi_{\widehat{B}}=\varphi_{\widehat{B}} \circ \hat{R}$ on $\widehat{B}$. Using (2.17), we see that $\psi_{\widehat{B}}$ is right invariant. Therefore $(\widehat{B}, \Delta)$ is a quantum group in the sense of [16]. Clearly the restriction $\left.\hat{\varepsilon}\right|_{\widehat{B}}$ is a normal counit on $\widehat{B}$. Hence $(\widehat{B}, \Delta)$ is a discrete quantum group. The counit $\left.\hat{\varepsilon}\right|_{\widehat{B}}$ is given by cutting elements at the Jones projection $e_{B}$ as follows.

Lemma 3.16. The equality $x e_{B}=\hat{\varepsilon}(x) e_{B}=e_{B} x$ holds for all $x \in \widehat{B}$. In particular, the Jones projection $e_{B}$ is a minimal central projection of $\widehat{B}$.

Proof. By Lemma 3.5, $\left(e_{B} \otimes 1\right) W_{\mathbb{G}}^{*}\left(1 \otimes e_{B}\right)=W_{\mathbb{G}}^{*}\left(e_{B} \otimes e_{B}\right)$. Then we have

$$
\Delta\left(e_{B}\right)\left(1 \otimes e_{B}\right)=W_{\mathbb{G}}\left(e_{B} \otimes 1\right) W_{\mathbb{G}}^{*}\left(1 \otimes e_{B}\right)=e_{B} \otimes e_{B} .
$$

Taking the adjoint of the above equality, we have

$$
\Delta\left(e_{B}\right)\left(1 \otimes e_{B}\right)=e_{B} \otimes e_{B}=\left(1 \otimes e_{B}\right) \Delta\left(e_{B}\right) .
$$

Take any $\omega \in L(\mathbb{G})_{*}$ and set $x=(\omega \otimes \mathrm{id})\left(\Delta\left(e_{B}\right)\right)$. Then we have

$$
\begin{aligned}
x e_{B} & =(\omega \otimes \mathrm{id})\left(\Delta\left(e_{B}\right)\right) e_{B}=(\omega \otimes \mathrm{id})\left(\Delta\left(e_{B}\right)\left(1 \otimes e_{B}\right)\right) \\
& =(\omega \otimes \mathrm{id})\left(e_{B} \otimes e_{B}\right)=\omega\left(e_{B}\right) e_{B}=\hat{\varepsilon}(x) e_{B} .
\end{aligned}
$$

Similarly we obtain $e_{B} x=\hat{\varepsilon}(x) e_{B}$. Then the desired equality holds for all $x \in \widehat{B}$ by Lemma 3.8 .

We summarize our arguments as follows.

Theorem 3.17. Let $B \subset L^{\infty}(\mathbb{G})$ be a right coideal. If $B$ has the expectation property and the coaction symmetry, then the pair $(\widehat{B}, \Delta)$ is a discrete quantum group.

Under assumption on co-amenability, we obtain the following characterization of right coideals of quotient type.

Theorem 3.18. Let $\mathbb{G}$ be a co-amenable compact quantum group and $B \subset L^{\infty}(\mathbb{G})$ a right coideal. Then $B$ is of quotient type if and only if $B$ has the expectation property and the coaction symmetry.

Proof. We have already proved the "only if" part in Lemma 3.2. So, it suffices to show the "if" part. By the previous theorem, $(\widehat{B}, \Delta)$ is a discrete quantum group. Let $\mathbb{H}=\left(C(\mathbb{H}), \delta_{\mathbb{H}}\right)$ be a compact quantum group such that the discrete quantum groups $\left(L(\mathbb{H}), \Delta_{\mathbb{H}}\right)$ and $(\widehat{B}, \Delta)$ are isomorphic. We identify $\widehat{B}$ with 
$L(\mathbb{H})$. By Lemma 2.10, $\mathbb{H}$ is represented as an algebraic quantum subgroup of $\mathbb{G}$, that is, there exists a restriction map $r_{\mathbb{H}}: A(\mathbb{G}) \rightarrow A(\mathbb{H})$ such that $r_{\mathbb{H}}^{t}: \widehat{B} \rightarrow L(\mathbb{G})$ is a given inclusion. Moreover by Lemma $2.8,\left\{\mathbb{H}, r_{\mathbb{H}}\right\}$ is in fact a quantum subgroup of $\mathbb{G}$. By Lemma 2.12 and Lemma 3.4, we have $B=\widehat{B}^{\prime} \cap L^{\infty}(\mathbb{G})=$ $L(\mathbb{H})^{\prime} \cap L^{\infty}(\mathbb{G})=L^{\infty}(\mathbb{H} \backslash \mathbb{G})$.

\section{Application to Classification of Poisson boundaries}

In the rest of this paper, we determine the Poisson boundary for a co-amenable compact quantum group with the commutative fusion rules. We also compute the Poisson boundary for a $q$-deformed classical compact Lie group.

\subsection{Poisson boundaries.}

We briefly recall the notion of the Poisson boundary for a discrete quantum group. We refer to [10, [12] and [17] for definitions of terminology.

Let $\phi_{\pi} \in B\left(H_{\pi}\right)_{*}$ be the right $\mathbb{G}$-invariant state. Define a transition operator $P_{\pi}$ on $R(\mathbb{G})$ by $P_{\pi}(x)=\left(\mathrm{id} \otimes \phi_{\pi}\right)\left(\Delta_{R}(x)\right)$ for $x \in R(\mathbb{G})$. For a probability measure $\mu$ on $\operatorname{Irr}(\mathbb{G})$, we set a non-commutative Markov operator

$$
P_{\mu}=\sum_{\pi \in \operatorname{Irr}(\mathbb{G})} \mu(\pi) P_{\pi}
$$

Then for a generating measure $\mu$, we define an operator system

$$
H^{\infty}\left(\widehat{\mathbb{G}}, P_{\mu}\right)=\left\{x \in R(\mathbb{G}) \mid P_{\mu}(x)=x\right\} .
$$

It has the von Neumann algebra structure defined by

$$
x \cdot y=\lim _{n \rightarrow \infty} P_{\mu}^{n}(x y) \quad \text { for } x, y \in H^{\infty}\left(\widehat{\mathbb{G}}, P_{\mu}\right),
$$

where the limit is taken in the strong topology [10, Theorem 3.6]. The von Neumann algebra $H^{\infty}\left(\widehat{\mathbb{G}}, P_{\mu}\right)$ is called the (non-commutative) Poisson boundary of $\left\{R(\mathbb{G}), P_{\mu}\right\}$. Assuming that the fusion algebra of $\mathbb{G}$ is commutative, we know that the Poisson boundary does not depend on the generating measure [12, Proposition 1.1], that is, we have

$$
H^{\infty}\left(\widehat{\mathbb{G}}, P_{\mu}\right)=\left\{x \in R(\mathbb{G}) \mid P_{\pi}(x)=x \text { for all } \pi \in \operatorname{Irr}(\mathbb{G})\right\} .
$$

We are interested only in such a compact quantum group, so we write simply $H^{\infty}(\widehat{\mathbb{G}})$ for $H^{\infty}\left(\widehat{\mathbb{G}}, P_{\mu}\right)$. The Poisson integral $\Theta: L^{\infty}(\mathbb{G}) \rightarrow R(\mathbb{G})$ is defined by

$$
\Theta=(\operatorname{id} \otimes h) \circ \beta,
$$

which maps $L^{\infty}(\mathbb{G})$ into $H^{\infty}(\widehat{\mathbb{G}})[10$, Lemma 3.8]. It is $\widehat{\mathbb{G}}-\mathbb{G}$-equivariant in the following sense:

$$
\alpha \circ \Theta=(\Theta \otimes \mathrm{id}) \circ \delta, \quad \Delta_{R} \circ \Theta=(\mathrm{id} \otimes \Theta) \circ \beta .
$$

Now we consider how the inverse map of $\Theta$ can be constructed. That will be written as a similar form to (4.1), that is, $\alpha$ and some state $\omega$ on $R(\mathbb{G})$ will take the place of $\beta$ and $h$, respectively. Then we consider the map $R(\mathbb{G}) \ni x \mapsto$ $(\omega \otimes \mathrm{id})(\alpha(x)) \in L^{\infty}(\mathbb{G})$. The inverse of $\Theta$ (if it exists) should be $\widehat{\mathbb{G}}$ - $\mathbb{G}$-equivariant 
as $\Theta$ is. Here, we have to realize what property of $h$ derives the bi-equivariance of $\Theta$ in the proof of [10, Lemma 3.8]. While the $\widehat{\mathbb{G}}$-equivariance follows by definition of the left action, the $\mathbb{G}$-equivariance does by right invariance of $h$. Hence the state $\omega$ has to satisfy the left invariance for the coproduct $\Delta_{R}$.

From now we assume the amenability of $\left(R(\mathbb{G}), \Delta_{R}\right)$. Let $m \in R(\mathbb{G})^{*}$ be a left invariant mean. Although $m$ is non-normal in general, we can consider a unital completely positive map $m \otimes \mathrm{id}: R(\mathbb{G}) \otimes M \rightarrow M$ for any von Neumann algebra $M$. Indeed for any $x \in R(\mathbb{G}) \otimes M$, we define an element $(m \otimes \mathrm{id})(x) \in M=$ $\left(M_{*}\right)^{*}$ by $\omega((m \otimes \mathrm{id})(x))=m((\mathrm{id} \otimes \omega)(x))$ for $\omega \in M_{*}$. Let $M$ and $N$ be von Neumann algebras and $T: M \rightarrow N$ a normal completely bounded map. Then $\left(m \otimes \mathrm{id}_{N}\right) \circ(\mathrm{id} \otimes T)=T \circ\left(m \otimes \mathrm{id}_{M}\right)$ holds. Here we need to assume the normalcy of $T$. In particular, we have $(m \otimes \mathrm{id})((1 \otimes a) x(1 \otimes b))=a(m \otimes \mathrm{id})(x) b$ for all $x \in R(\mathbb{G}) \otimes M$ and $a, b \in M$.

Now define a unital completely positive map $\Lambda: R(\mathbb{G}) \rightarrow L^{\infty}(\mathbb{G})$ by

$$
\Lambda(x)=(m \otimes \mathrm{id})(\alpha(x)) \text { for } x \in R(\mathbb{G}) .
$$

It turns out that $\Lambda$ is normal on the Poisson boundary $H^{\infty}(\widehat{\mathbb{G}})$ in the next lemma. Moreover by Theorem 4.8, we will see that $\Lambda$ is actually equal to $\Theta^{*}$ defined in [12]. This means that $\Lambda$ does not depend on the choice of an left invariant mean.

Lemma 4.1. The following statements hold.

(1) $h \circ \Lambda=\hat{\varepsilon}$ on $H^{\infty}(\widehat{\mathbb{G}})$. In particular, $\Lambda$ is a faithful normal map.

(2) $\Lambda$ is $\widehat{\mathbb{G}}-\mathbb{G}$-equivariant, that is,

$$
\delta \circ \Lambda=(\Lambda \otimes \mathrm{id}) \circ \alpha, \quad \beta \circ \Lambda=(\operatorname{id} \otimes \Lambda) \circ \Delta_{R} .
$$

Proof. (1). Since the fusion algebra of $\mathbb{G}$ is commutative, the action $\alpha$ on $H^{\infty}(\widehat{\mathbb{G}})$ is ergodic [10, Corollary 3.7]. Hence $E_{\alpha}(x)=\hat{\varepsilon}(x) 1$ for all $x \in H^{\infty}(\widehat{\mathbb{G}})$. Then $h(\Lambda(x))=m\left(E_{\alpha}(x)\right)=m(\hat{\varepsilon}(x) 1)=\hat{\varepsilon}(x)$. Since $h$ and $\hat{\varepsilon}$ are faithful normal states [10, Theorem 3.6], we conclude that $\Lambda$ is a faithful normal map.

(2). First we show the $\mathbb{G}$-equivariance of $\Lambda$. Let $x \in R(\mathbb{G})$. Then we have

$$
\begin{aligned}
\delta(\Lambda(x)) & =\delta((m \otimes \mathrm{id})(\alpha(x)))=(m \otimes \mathrm{id} \otimes \mathrm{id})((\mathrm{id} \otimes \delta)(\alpha(x))) \\
& =(m \otimes \mathrm{id} \otimes \mathrm{id})((\alpha \otimes \mathrm{id})(\alpha(x))) \\
& =((m \otimes \mathrm{id}) \circ \alpha \otimes \mathrm{id})(\alpha(x))=(\Lambda \otimes \mathrm{id})(\alpha(x)) .
\end{aligned}
$$

Next we show the $\widehat{\mathbb{G}}$-equivariance of $\Lambda$. The left invariance of $m$ yields $($ id $\otimes m)\left(\Delta_{R}(y)\right)=m(y) 1$ for all $y \in R(\mathbb{G})$. Take any $x \in R(\mathbb{G})$ and then

$$
\begin{aligned}
& (\mathrm{id} \otimes \Lambda)\left(\Delta_{R}(x)\right) \\
= & (\mathrm{id} \otimes m \otimes \mathrm{id})\left((\mathrm{id} \otimes \alpha)\left(\Delta_{R}(x)\right)\right) \\
= & (\mathrm{id} \otimes m \otimes \mathrm{id})\left(\left(V_{\mathbb{G}}\right)_{23}\left(V_{\mathbb{G}}\right)_{12}^{*}(1 \otimes x \otimes 1)\left(V_{\mathbb{G}}\right)_{12}\left(V_{\mathbb{G}}\right)_{23}^{*}\right) \\
= & (\mathrm{id} \otimes m \otimes \mathrm{id})\left(\left(V_{\mathbb{G}}\right)_{13}^{*}\left(V_{\mathbb{G}}\right)_{12}^{*}\left(V_{\mathbb{G}}\right)_{23}(1 \otimes x \otimes 1)\left(V_{\mathbb{G}}\right)_{23}^{*}\left(V_{\mathbb{G}}\right)_{12}\left(V_{\mathbb{G}}\right)_{13}\right) \\
= & V_{\mathbb{G}}^{*}(\mathrm{id} \otimes m \otimes \mathrm{id})\left(\left(\Delta_{R} \otimes \mathrm{id}\right)(\alpha(x))\right) V_{\mathbb{G}} \\
= & V_{\mathbb{G}}^{*}\left((\mathrm{id} \otimes m) \circ \Delta_{R} \otimes \mathrm{id}\right)(\alpha(x)) V_{\mathbb{G}} \\
& \quad{ }_{22}
\end{aligned}
$$




$$
=V_{\mathbb{G}}^{*}(1 \otimes(m \otimes \mathrm{id})(\alpha(x))) V_{\mathbb{G}}=\beta(\Lambda(x))
$$

Lemma 4.2. For any $x \in H^{\infty}(\widehat{\mathbb{G}}), \Theta(\Lambda(x))=x$. In particular, $\Lambda$ is a faithful normal *-homomorphism.

Proof. Let $x \in H^{\infty}(\widehat{\mathbb{G}})$. By Lemma 4.1, we have

$$
\begin{aligned}
\Theta(\Lambda(x)) & =(\mathrm{id} \otimes h)(\beta(\Lambda(x)))=(\mathrm{id} \otimes h)\left((\mathrm{id} \otimes \Lambda)\left(\Delta_{R}(x)\right)\right) \\
& =(\mathrm{id} \otimes \hat{\varepsilon})\left(\Delta_{R}(x)\right)=x .
\end{aligned}
$$

The multiplicativity of $\Lambda$ is shown as follows. For any $x \in H^{\infty}(\widehat{\mathbb{G}})$, we have

$$
\begin{aligned}
x^{*} \cdot x & =\Theta(\Lambda(x))^{*} \cdot \Theta(\Lambda(x)) \leq \Theta\left(\Lambda(x)^{*} \Lambda(x)\right) \\
& \leq \Theta\left(\Lambda\left(x^{*} \cdot x\right)\right)=x^{*} \cdot x .
\end{aligned}
$$

Hence we obtain $\Theta\left(\Lambda(x)^{*} \Lambda(x)\right)=\Theta\left(\Lambda\left(x^{*} \cdot x\right)\right)$, namely, $\Lambda(x)^{*} \Lambda(x)=\Lambda\left(x^{*} \cdot x\right)$. This implies that $\Lambda$ is a $*$-homomorphism.

We determine the multiplicative domain of $\Theta$.

Lemma 4.3. Set $B=\Lambda\left(H^{\infty}(\widehat{\mathbb{G}})\right)$.

(1) $B$ is a right coideal with the expectation property and the coaction symmetry.

(2) The conditional expectation onto $B$ is $\widehat{\mathbb{G}}$-equivariant.

(3) $B$ coincides with the multiplicative domain of $\Theta$. Moreover, the Poisson integral $\Theta: B \rightarrow H^{\infty}(\widehat{\mathbb{G}})$ is an isomorphism.

Proof. (1). By the previous lemma, $B \subset L^{\infty}(\mathbb{G})$ is a von Neumann subalgebra. Since $\Lambda$ is $\widehat{\mathbb{G}}-\mathbb{G}$-equivariant by Lemma 4.1, $B$ is globally invariant under the actions $\alpha$ and $\beta$. Hence $B$ is a right coideal with the coaction symmetry. Set $E_{B}=\Lambda \circ \Theta$. Then by the previous lemma, we see that $E_{B}$ is a faithful normal conditional expectation onto $B$. For $x \in L^{\infty}(\mathbb{G})$, we have

$$
h\left(E_{B}(x)\right)=h(\Lambda(\Theta(x)))=\hat{\varepsilon}(\Theta(x))=h(x) .
$$

Hence $B$ has the expectation property.

(2). Since $\Theta$ and $\Lambda$ are $\widehat{\mathbb{G}}$-equivariant, $E_{B}=\Theta \circ \Lambda$ is also $\widehat{\mathbb{G}}$-equivariant.

(3). Let $\mathcal{D} \subset L^{\infty}(\mathbb{G})$ be the multiplicative domain of $\Theta$. It is easy to see that $B \subset \mathcal{D}$. We show the converse inclusion. Let $x \in \mathcal{D}$. Then by definition, we have $\Theta\left(x^{*} x\right)=\Theta(x)^{*} \cdot \Theta(x)$ and $\Theta\left(x x^{*}\right)=\Theta(x) \cdot \Theta(x)^{*}$. Applying $\Lambda$ to both the sides of the equalities, we have $E_{B}\left(x^{*} x\right)=E_{B}(x)^{*} E_{B}(x)$ and $E_{B}\left(x x^{*}\right)=E_{B}(x) E_{B}(x)^{*}$. This immediately yields that $x \in B$, and $B=\mathcal{D}$. Hence $\Theta: B \rightarrow H^{\infty}(\widehat{\mathbb{G}})$ is a faithful normal $*$-homomorphism. The surjectivity of $\Theta$ follows from $\Theta(B)=$ $\Theta\left(\Lambda\left(H^{\infty}(\widehat{\mathbb{G}})\right)\right)=H^{\infty}(\widehat{\mathbb{G}})$.

Lemma 4.4. Let $\mathbb{H}$ be a quantum subgroup of $\mathbb{G}$. Then $\mathbb{H}$ is of Kac type if and only if the expectation $E_{\mathbb{H}}: L^{\infty}(\mathbb{G}) \rightarrow L^{\infty}(\mathbb{H} \backslash \mathbb{G})$ is $\widehat{\mathbb{G}}$-equivariant, that is, it satisfies $\left(\mathrm{id} \otimes E_{\mathbb{H}}\right) \circ \beta=\beta \circ E_{\mathbb{H}}$. 
Proof. For any $x \in C(\mathbb{G})$, we have

$$
\begin{aligned}
\left(\mathrm{id} \otimes E_{\mathbb{H}}\right)(\beta(x)) & =\left(\mathrm{id} \otimes h_{\mathbb{H}} \circ r_{\mathbb{H}} \otimes \mathrm{id}\right)\left((\mathrm{id} \otimes \delta)\left(V_{\mathbb{G}}^{*}(1 \otimes x) V_{\mathbb{G}}\right)\right) \\
& =\left(\mathrm{id} \otimes h_{\mathbb{H}} \circ r_{\mathbb{H}} \otimes \mathrm{id}\right)\left(\left(V_{\mathbb{G}}\right)_{13}^{*}\left(V_{\mathbb{G}}\right)_{12}^{*}(1 \otimes \delta(x))\left(V_{\mathbb{G}}\right)_{12}\left(V_{\mathbb{G}}\right)_{13}\right) \\
& =V_{\mathbb{G}}^{*}\left(\mathrm{id} \otimes h_{\mathbb{H}} \otimes \mathrm{id}\right)\left(\left(V_{\mathbb{H}}\right)_{12}^{*}\left(1 \otimes\left(r_{\mathbb{H}} \otimes \mathrm{id}\right)(\delta(x))\right)\left(V_{\mathbb{H}}\right)_{12}\right) V_{\mathbb{G}} .
\end{aligned}
$$

This is equal to $\beta\left(E_{\mathbb{H}}(x)\right)=V_{\mathbb{G}}^{*}\left(1 \otimes E_{\mathbb{H}}(x)\right) V_{\mathbb{G}}$ if and only if $\left(\mathrm{id} \otimes h_{\mathbb{H}} \otimes \mathrm{id}\right)\left(\left(V_{\mathbb{H}}\right)_{12}^{*}\left(1 \otimes\left(r_{\mathbb{H}} \otimes \mathrm{id}\right)(\delta(x))\right)\left(V_{\mathbb{H}}\right)_{12}\right)=1 \otimes\left(h_{\mathbb{H}} \otimes \mathrm{id}\right)\left(\left(r_{\mathbb{H}} \otimes \mathrm{id}\right)(\delta(x))\right)$. Multiplying $1 \otimes 1 \otimes y, y \in C(\mathbb{G})$ from the right, we have

$$
\begin{aligned}
& \left(\mathrm{id} \otimes h_{\mathbb{H}} \otimes \mathrm{id}\right)\left(\left(V_{\mathbb{H}}\right)_{12}^{*}\left(1 \otimes\left(r_{\mathbb{H}} \otimes \mathrm{id}\right)(\delta(x)(1 \otimes y))\right)\left(V_{\mathbb{H}}\right)_{12}\right) \\
= & 1 \otimes\left(h_{\mathbb{H}} \otimes \mathrm{id}\right)\left(\left(r_{\mathbb{H}} \otimes \mathrm{id}\right)(\delta(x)(1 \otimes y))\right) .
\end{aligned}
$$

Since the subspace $\delta(C(\mathbb{G}))(\mathbb{C} \otimes C(\mathbb{G}))$ is dense in $C(\mathbb{G}) \otimes C(\mathbb{G})$, we have for all $x \in C(\mathbb{G}) \otimes C(\mathbb{G})$,

$\left.\left(\mathrm{id} \otimes h_{\mathbb{H}} \otimes \mathrm{id}\right)\left(\left(V_{\mathbb{H}}\right)_{12}^{*}\left(1 \otimes\left(r_{\mathbb{H}} \otimes \mathrm{id}\right)(x)\right)\left(V_{\mathbb{H}}\right)_{12}\right)=1 \otimes\left(h_{\mathbb{H}} \otimes \mathrm{id}\right)\left(\left(r_{\mathbb{H}} \otimes \mathrm{id}\right)(x)\right)\right)$.

More precisely, we have

$$
\left(\mathrm{id} \otimes h_{\mathbb{H}}\right)\left(V_{\mathbb{H}}^{*}(1 \otimes z) V_{\mathbb{H}}\right)=h_{\mathbb{H}}(z) 1 \quad \text { for all } z \in C(\mathbb{H}) .
$$

This shows that $E_{\mathbb{H}}$ is $\widehat{\mathbb{G}}$-equivariant if and only if the image of the Poisson integral of $\mathbb{H}$ consists of scalars. By [10, Corollary 3.9], it is equivalent to that $\mathbb{H}$ is of Kac type.

Lemma 4.5. Let $\mathbb{K}$ be a quantum subgroup of Kac type in $\mathbb{G}$. Then $\Theta \circ E_{\mathbb{K}}=\Theta$.

Proof. By Lemma 4.4, $E_{\mathbb{K}}$ is $\widehat{\mathbb{G}}$-equivariant. For any $x \in L^{\infty}(\mathbb{G})$, we have

$$
\begin{aligned}
\Theta\left(E_{\mathbb{K}}(x)\right) & =(\mathrm{id} \otimes h)\left(\beta\left(E_{\mathbb{K}}(x)\right)\right)=(\mathrm{id} \otimes h)\left(\left(\mathrm{id} \otimes E_{\mathbb{K}}\right)(\beta(x))\right) \\
& =(\mathrm{id} \otimes h)(\beta(x))=\Theta(x) .
\end{aligned}
$$

We prepare the notion of maximality for a quantum subgroup of Kac type as follows.

Definition 4.6. Let $\mathbb{G}$ be a compact quantum group. We say that a quantum subgroup $\mathbb{H}$ of Kac type is maximal if for any quantum subgroup $\mathbb{K}$ of Kac type, we have $L^{\infty}(\mathbb{H} \backslash \mathbb{G}) \subset L^{\infty}(\mathbb{K} \backslash \mathbb{G})$.

Lemma 4.7. Let $\mathbb{G}$ be a compact quantum subgroup. If there exists a maximal quantum subgroup of Kac type, it is unique in the following sense. Let $\left\{\mathbb{H}, r_{\mathbb{H}}\right\}$ and $\left\{\mathbb{K}, r_{\mathbb{K}}\right\}$ be maximal quantum subgroups of Kac type. Then there exists a *-isomorphism $\theta: C(\mathbb{H}) \rightarrow C(\mathbb{K})$ such that $r_{\mathbb{K}}=\theta \circ r_{\mathbb{H}}$.

Proof. By definition, we have $L^{\infty}(\mathbb{H} \backslash \mathbb{G})=L^{\infty}(\mathbb{K} \backslash \mathbb{G})$. Then by Lemma 2.13, we have the desired $*$-isomorphism.

Theorem 4.8. Let $\mathbb{G}$ be a co-amenable compact quantum group. Assume that its fusion algebra is commutative. Then the following statements hold. 
(1) There exists a unique maximal quantum subgroup of Kac type $\mathbb{H}$.

(2) The Poisson integral $\Theta: L^{\infty}(\mathbb{H} \backslash \mathbb{G}) \rightarrow H^{\infty}(\widehat{\mathbb{G}})$ is an isomorphism.

Proof. (1). By using Theorem 3.18, Lemma 4.4 and Lemma 4.3, there exists a quantum subgroup $\mathbb{H}$ of Kac type such that $B=L^{\infty}(\mathbb{H} \backslash \mathbb{G})$. We show the maximality of $\mathbb{H}$. Let $\mathbb{K}$ be another quantum subgroup of Kac type. By Lemma 4.5, $\Theta \circ E_{\mathbb{K}}=\Theta$. Since $E_{B}=\Lambda \circ \Theta$, we have $E_{B} \circ E_{\mathbb{K}}=E_{B}$. Let $e_{\mathbb{K}}$ be the Jones projection associated with $E_{\mathbb{K}}$. Then $e_{B} e_{\mathbb{K}}=e_{B}$, and hence $e_{\mathbb{K}} e_{B}=e_{B}$. It yields $B \subset L^{\infty}(\mathbb{K} \backslash \mathbb{G})$. Hence $\mathbb{H}$ is maximal.

(2). It follows from Lemma 4.3 (3).

Remark 4.9. We have realized the existence of the maximal quantum subgroup of Kac type by studying the Poisson integral. However, the notion has been already introduced in [20, Appendix A], where it is called the canonical Kac quotient. The canonical Kac quotient is the function algebra on the maximal quantum subgroup of Kac type. The existence is proved for an arbitrary compact quantum group [20, Proposition A.1].

\section{2. q-deformed classical compact Lie groups.}

We determine the maximal quantum subgroup of Kac type in a $q$-deformed classical compact Lie group $(0<q<1)$. In order to do so, we freely make use of terminology and results in [15] such as construction of quantum universal enveloping algebras, quantized function algebras and so on.

Let $\mathfrak{g}$ be a complex classical simple Lie algebra with the simple roots $\left\{\alpha_{i}\right\}_{i=1}^{n}$. We denote by $\mathbb{G}$ the corresponding classical compact Lie group [15, Definition 1.2.4 of Chapter 3]. Let $U_{q}(\mathfrak{g})$ be the quantum universal enveloping algebra associated with $\mathfrak{g}$ [15, Definition 7.1.1 of Chapter 2]. We equip $U_{q}(\mathfrak{g})$ with the Hopf $*$-algebra structure as in [15, Proposition 1.2.3 of Chapter 3]. We denote by $W$ the Weyl group and by $s_{i}$ the simple reflection with respect to $\alpha_{i}$.

For a dominant integral weight $\lambda$, we denote by $L_{q}(\lambda)$ the irreducible $U_{q}(\mathfrak{g})$ module with the highest weight $\lambda$. Fix an orthonormal basis $\left\{\xi_{\mu}^{r}\right\}_{r}$ of each weight space $L_{q}(\lambda)_{\mu}$ with the weight $\mu$. We define $C_{\xi_{\mu}^{r}, \xi_{\nu}^{s}}^{\lambda} \in A(\mathbb{G}) \subset U_{q}(\mathfrak{g})^{*}$ by

$$
C_{\xi_{\mu}^{r}, \xi_{\nu}^{s}}^{\lambda}(x)=\left(x \xi_{\nu}^{s}, \xi_{\mu}^{r}\right) \quad \text { for } x \in U_{q}(\mathfrak{g}) .
$$

Then we have $A\left(\mathbb{G}_{q}\right)=\operatorname{span}\left\{C_{\xi_{\mu}^{r}, \xi_{\nu}^{s}}^{\lambda}\right\}_{\lambda, \mu, \nu, r, s}$. On the involution, we have

$$
\left(C_{\xi_{\mu}^{r}, \xi_{\nu}^{s}}^{\lambda}\right)^{*}=q^{(\mu-\nu, \rho)} C_{\xi_{-\mu}^{r}, \xi_{-\nu}^{s}}^{-w_{0} \lambda},
$$

where $\varrho=(1 / 2) \sum_{i=1}^{n} \alpha_{i}$ is the Weyl vector and $w_{0} \in W$ is the longest element. Let $\kappa$ and $\left\{\tau_{t}\right\}_{t \in \mathbb{R}}$ be the antipode and the scaling automorphism group of $\mathbb{G}_{q}$ introduced in $\$ 2.1$. From (2.5), $\kappa^{2}=\tau_{-i}$ and (4.2), we obtain

$$
\tau_{t}\left(C_{\xi_{\mu}^{r}, \xi_{\nu}^{s}}^{\lambda}\right)=q^{i(\mu-\nu, 2 \varrho) t} C_{\xi_{\mu}^{r}, \xi_{\nu}^{s}}^{\lambda} .
$$

We denote by $C\left(\mathbb{G}_{q}\right)$ the $C^{*}$-completion of $A\left(\mathbb{G}_{q}\right)$ with respect to the universal norm. Set $q_{i}=q^{\left(\alpha_{i}, \alpha_{i}\right) / 2}, i=1, \ldots, n$. Let $U_{q_{i}}(s u(2))$ be the quantized universal enveloping algebra of the Lie algebra $s u(2)$ with the deformation parameter 
$q_{i}$. The canonical embedding $U_{q_{i}}(s u(2)) \rightarrow U_{q}(\mathfrak{g})$ induces the restriction map $r_{i}: A\left(\mathbb{G}_{q}\right) \rightarrow A\left(S U_{q_{i}}(2)\right)$ [15, Subsection 6.1 of Chapter 3]. Since $C\left(\mathbb{G}_{q}\right)$ is a universal $C^{*}$-algebra, $r_{i}$ extends to the $*$-homomorphism $C\left(\mathbb{G}_{q}\right) \rightarrow C\left(S U_{q_{i}}(2)\right)$. Take a canonical infinite dimensional irreducible representation $\pi_{i}: C\left(S U_{q_{i}}(2)\right) \rightarrow$ $B\left(\ell_{2}\right)$ defined in [15, Proposition 4.1.1 of Chapter 3]. We note that the counit $\varepsilon_{i}$ of $C\left(S U_{q_{i}}(2)\right)$ factors through $\operatorname{Im}\left(\pi_{i}\right)$, that is, there exists a $*$-homomorphism $\eta_{i}: \operatorname{Im}\left(\pi_{i}\right) \rightarrow \mathbb{C}$ such that $\eta_{i} \circ \pi_{i}=\varepsilon_{i}$. Indeed, let $p: B\left(\ell_{2}\right) \rightarrow Q$ be the canonical surjection onto the Calkin algebra $Q$. Let $S \in B\left(\ell_{2}\right)$ be the unilateral shift. By definition of $\pi_{i}$, we see that $p\left(\operatorname{Im}\left(\pi_{i}\right)\right)$ is a commutative $C^{*}$-algebra generated by a unitary $p(S)$. Take the character $\omega: p\left(\operatorname{Im}\left(\pi_{i}\right)\right) \rightarrow \mathbb{C}$ defined by $\omega(p(S))=1$. Then the character $\eta_{i}:=\omega \circ p: \operatorname{Im}\left(\pi_{i}\right) \rightarrow \mathbb{C}$ has the desired property.

Let $\left\{\mathbb{T}, r_{\mathbb{T}}\right\}$ be the maximal torus subgroup of $\mathbb{G}_{q}$, where $r_{\mathbb{T}}: C\left(\mathbb{G}_{q}\right) \rightarrow C(\mathbb{T})$ is a restriction map. Any one dimensional $*$-homomorphism of $C\left(\mathbb{G}_{q}\right)$ is given by $\pi_{t}:=\chi_{t} \circ r_{\mathbb{T}}$, where $\chi_{t}: C(\mathbb{T}) \rightarrow \mathbb{C}$ is the evaluation at $t \in \mathbb{T}$. Then for an element $w \in W \backslash\{e\}$ with a reduced decomposition $w=s_{i_{1}} \cdots s_{i_{k}}$ and $t \in \mathbb{T}$, we define

$$
\pi_{w, t}=\left(\pi_{i_{1}} \circ r_{i_{1}} \otimes \ldots \otimes \pi_{i_{k}} \circ r_{i_{k}} \otimes \pi_{t}\right) \circ \delta_{\mathbb{G}_{q}}^{(k)},
$$

where $\delta_{\mathbb{G}_{q}}^{(k)}: C\left(\mathbb{G}_{q}\right) \rightarrow C\left(\mathbb{G}_{q}\right)^{\otimes(k+1)}$ is recursively defined by $\delta_{\mathbb{G}_{q}}^{(k)}=\left(\delta_{\mathbb{G}_{q}} \otimes \mathrm{id}^{\otimes(k-1)}\right) \circ$ $\delta_{\mathbb{G}_{q}}^{(k-1)}$ and $\delta_{\mathbb{G}_{q}}^{(1)}=\delta_{\mathbb{G}_{q}}$. Then $\pi_{w, t}$ is an irreducible $*$-homomorphism of $C\left(\mathbb{G}_{q}\right)$ and does not depend on the choice of the reduced decomposition of $w$ up to equivalence [15, Theorem 6.2.1 of Chapter 3]. Every irreducible $*$-homomorphism of $C\left(\mathbb{G}_{q}\right)$ is equivalent to some $\pi_{w, t}[15$, Theorem 6.2.7 of Chapter 3].

Lemma 4.10. Let $\mathbb{G}_{q}$ be the q-deformation of a classical compact Lie group $\mathbb{G}$. Then its maximal quantum subgroup of Kac type is the maximal torus $\mathbb{T}$.

Proof. Let $\left\{\mathbb{H}, r_{\mathbb{H}}\right\}$ be a quantum subgroup of Kac type. We will show that $\mathbb{H}$ is a subgroup of $\mathbb{T}$. Assume that $C(\mathbb{H})$ is not commutative. Then there exists an irreducible $*$-homomorphism $\rho: C(\mathbb{H}) \rightarrow B\left(H_{\rho}\right)$ with $\operatorname{dim} H_{\rho} \geq 2$. Set $\pi=\rho \circ r_{\mathbb{H}}$. We may assume $\pi=\pi_{w, t}$ for some element $w \in W \backslash\{e\}$ with a reduced expression $w=s_{i_{1}} \cdots s_{i_{k}}$ and $t \in \mathbb{T}$. Then we consider the irreducible $*$-homomorphism (id $\left.\otimes \eta_{i_{2}} \cdots \otimes \eta_{i_{k}}\right) \circ \pi=\left(\pi_{i_{1}} \circ r_{i_{1}} \otimes \pi_{t}\right) \circ \delta_{\mathbb{G}_{q}}=\pi_{s_{i_{1}}, t}$, which factors through $C(\mathbb{H})$. Hence we may assume $\pi=\rho \circ r_{\mathbb{H}}=\pi_{s_{j}, t}$ for some $1 \leq j \leq n$ and $t \in \mathbb{T}$. Since the scaling automorphism of $C(\mathbb{H})$ is trivial, we have $r_{\mathbb{H}} \circ \tau_{t}=r_{\mathbb{H}}$ for all $t \in \mathbb{R}$ by Lemma 2.9. Let $\omega_{j}$ be the fundamental weight for $\alpha_{j}$. Setting

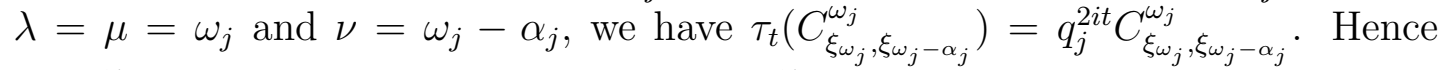
$r_{\mathbb{H}}\left(C_{\xi_{\omega_{j}}, \xi_{\omega_{j}-\alpha_{j}}}^{\omega_{j}}\right)=0$. In particular, $\pi_{s_{j}, t}\left(C_{\xi_{\omega_{j}}, \xi_{\omega_{j}-\alpha_{j}}}^{\omega_{j}}\right)=0$. This is, however, in contradiction with $\pi_{s_{j}, t}\left(C_{\xi_{\omega_{j}}, \xi_{\omega_{j}-\alpha_{j}}^{\omega_{j}}}\right) \neq 0$ which is shown by direct computation.

Therefore $C(\mathbb{H})$ is a commutative $C^{*}$-algebra, that is, $\mathbb{H}$ is an ordinary compact group. For $x \in \mathbb{H}$, we write $\chi_{x}^{\mathbb{H}}$ for the character $*$-homomorphism. For $t \in \mathbb{T}$, we also use $\chi_{t}^{\mathbb{T}}$ as well. By the above arguments, for any $x \in \mathbb{H}$ there exists an element $t(x) \in \mathbb{T}$ such that $\chi_{x}^{\mathbb{H}} \circ r_{\mathbb{H}}=\chi_{t(x)}^{\mathbb{T}} \circ r_{\mathbb{T}}$. This shows that the map $t: \mathbb{H} \rightarrow \mathbb{T}$ 
is continuous. For any $x, y \in \mathbb{H}$, we have

$$
\begin{aligned}
\chi_{t(x y)}^{\mathbb{T}} \circ r_{\mathbb{T}} & =\chi_{x y}^{\mathbb{H}} \circ r_{\mathbb{H}}=\left(\chi_{x}^{\mathbb{H}} \otimes \chi_{y}^{\mathbb{H}}\right) \circ \delta_{\mathbb{H}} \circ r_{\mathbb{H}}=\left(\chi_{x}^{\mathbb{H}} \otimes \chi_{y}^{\mathbb{H}}\right) \circ\left(r_{\mathbb{H}} \otimes r_{\mathbb{H}}\right) \circ \delta_{\mathbb{G}_{q}} \\
& =\left(\chi_{t(x)}^{\mathbb{T}} \otimes \chi_{t(y)}^{\mathbb{T}}\right) \circ\left(r_{\mathbb{T}}^{\mathbb{T}} \otimes r_{\mathbb{T}}\right) \circ \delta_{\mathbb{G}_{q}}=\left(\chi_{t(x)}^{\mathbb{T}} \otimes \chi_{t(y)}^{\mathbb{T}}\right) \circ \delta_{\mathbb{T}} \circ r_{\mathbb{T}} \\
& =\chi_{t(x) t(y)}^{\mathbb{T}} \circ r_{\mathbb{T}} .
\end{aligned}
$$

Hence the map $t: \mathbb{H} \rightarrow \mathbb{T}$ is a group homomorphism. Next we show that $t$ is injective. For $x \in \mathbb{H}$, suppose that $\chi_{x}^{\mathbb{H}} \circ r_{\mathbb{H}}=\chi_{e}^{\mathbb{T}} \circ r_{\mathbb{T}}$ holds. Since it is equal to the counit $\varepsilon$ of $C\left(\mathbb{G}_{q}\right)$ by Lemma 2.9, we have

$$
\chi_{x}^{\mathbb{H}} \circ r_{\mathbb{H}}=\chi_{e}^{\mathbb{T}} \circ r_{\mathbb{T}}=\varepsilon=\chi_{e}^{\mathbb{H}} \circ r_{\mathbb{H}} .
$$

Hence $x=e$, and the map $t$ is injective. Since the left action of $x \in \mathbb{H}$ is given by $\left(\chi_{x}^{\mathbb{H}} \circ r_{\mathbb{H}} \otimes \mathrm{id}\right) \circ \delta_{\mathbb{G}_{q}}=\left(\chi_{t(x)}^{\mathbb{T}} \circ r_{\mathbb{T}} \otimes \mathrm{id}\right) \circ \delta_{\mathbb{G}_{q}}$, we have $L^{\infty}\left(\mathbb{T} \backslash \mathbb{G}_{q}\right) \subset L^{\infty}\left(\mathbb{H} \backslash \mathbb{G}_{q}\right)$. Therefore $\mathbb{T}$ is maximal.

By Theorem 4.8 and the previous lemma, we obtain the following corollary.

Corollary 4.11. Let $\mathbb{G}_{q}$ be the q-deformation of a classical compact Lie group $\mathbb{G}$. Then the Poisson integral $\Theta: L^{\infty}\left(\mathbb{T} \backslash \mathbb{G}_{q}\right) \rightarrow H^{\infty}\left(\widehat{\mathbb{G}_{q}}\right)$ is an isomorphism.

Acknowledgments. The author is grateful to Yasuyuki Kawahigashi and Masaki Izumi for encouragement and various useful comments. He would also like to thank Stefaan Vaes for informing him of canonical Kac quotients introduced in [20].

\section{REFERENCES}

[1] S. Baaj and G. Skandalis, Unitaires multiplicatifs et dualité pour les produits croisés de $C^{*}$-algèbres, Ann. Sci. École Norm. Sup. (4) 26 (1993), no. 4, 425-488.

[2] E. Bédos, R. Conti and L. Tuset, On amenability and co-amenability of algebraic quantum groups and their corepresentations, Canad. J. Math. 57 (2005), no. 1, 17-60.

[3] E. Bédos, G. Murphy and L. Tuset, Co-amenability of compact quantum groups, J. Geom. Phys. 40 (2001), no. 2, 130-153.

[4] E. Bédos, G. Murphy and L. Tuset, Amenability and coamenability of algebraic quantum groups, Int. J. Math. Math. Sci. 31 (2002), no. 10, 577-601.

[5] A. Connes, On the spatial theory of von Neumann algebras, J. Funct. Anal. 35 (1980), no. $2,153-164$.

[6] E. Effros and Z.-J. Ruan, Discrete quantum groups. I. The Haar measure, Internat. J. Math. 5 (1994), no. 5, 681-723.

[7] M. Enock and J. M. Schwartz, Kac algebras and duality of locally compact groups, SpringerVerlag, Berlin, 1992. x+257 pp.

[8] U. Haagerup, Operator-valued weights in von Neumann algebras. I, J. Funct. Anal. 32 (1979), no. 2, 175-206.

[9] U. Haagerup, Operator-valued weights in von Neumann algebras. II, J. Funct. Anal. 33 (1979), no. 3, 339-361.

[10] M. Izumi, Non-commutative Poisson boundaries and compact quantum group actions, Adv. Math. 169 (2002), no. 1, 1-57.

[11] M. Izumi, R. Longo and S. Popa, A Galois correspondence for compact groups of automorphisms of von Neumann algebras with a generalization to Kac algebras, J. Funct. Anal. 155 (1998), no. 1, 25-63. 
[12] M. Izumi, S. Neshveyev and L. Tuset, Poisson boundary of the dual of $\mathrm{SU}_{q}(n)$, Comm. Math. Phys. 262 (2006), no. 2, 505-531.

[13] H. Kosaki, Extension of Jones' theory on index to arbitrary factors, J. Funct. Anal. 66 (1986), no. 1, 123-140.

[14] H. Kosaki, Type III factors and index theory, Lecture Notes Series 43, Seoul National University, Research Institute of Mathematics, Global Analysis Research Center, Seoul, 1998. ii+96 pp.

[15] L. I. Korogodski and Y. S. Soibelman, Algebras of functions on quantum groups. Part I, Mathematical Surveys and Monographs 56, American Mathematical Society, Providence, RI, 1998. $\mathrm{x}+150 \mathrm{pp}$.

[16] J. Kustermans and S. Vaes, Locally compact quantum groups in the von Neumann algebraic setting, Math. Scand. 92 (2003), no. 1, 68-92.

[17] S. Neshveyev and L. Tuset, The Martin boundary of a discrete quantum group, J. Reine Angew. Math. 568 (2004), 23-70.

[18] P. Podleś, Quantum spheres, Lett. Math. Phys. 14 (1987), no. 3, 193-202.

[19] P. Podleś, Symmetries of quantum spaces. Subgroups and quotient spaces of quantum $\mathrm{SU}(2)$ and $\mathrm{SO}(3)$ groups, Comm. Math. Phys. 170 (1995), no. 1, 1-20.

[20] P. M. Soltan, Quantum Bohr compactification, Illinois J. Math. 49 (2005), no. 4, 12451270.

[21] M. Takesaki, Conditional expectations in von Neumann algebras, J. Funct. Anal. 9 (1972), $306-321$.

[22] R. Tomatsu, Amenable discrete quantum groups, J. Math. Soc. Japan 58 (2006), no. 4, 949-964.

[23] R. Tomatsu, Compact quantum ergodic systems, ArXiv:math.OA/0412012.

[24] S. Vaes, The unitary implementation of a locally compact quantum group action, J. Funct. Anal. 180 (2001), no. 2, 426-480.

[25] S. Vaes and N. Vander Vennet, Identification of the Poisson and Martin boundaries of orthogonal discrete quantum groups, ArXiv:math.OA/0605489.

[26] S. Vaes and R. Vergnioux, The boundary of universal discrete quantum groups, exactness and factoriality, ArXiv:math.OA/0509706

[27] A. Van Daele, Discrete quantum groups, J. Algebra 180 (1996), no. 2, 431-444.

[28] S. L. Woronowicz, Compact matrix pseudogroups, Comm. Math. Phys. 111 (1987), no. 4, 613-665.

[29] S. L. Woronowicz, Tannaka-Kreĭn duality for compact matrix pseudogroups. Twisted $\mathrm{SU}(N)$ groups, Invent. Math. 93 (1988), no. 1, 35-76.

[30] S. L. Woronowicz, Compact quantum groups, Symétries quantiques (Les Houches, 1995), 845-884, North-Holland, Amsterdam, (1998).

[31] S. Yamagami, On unitary representation theories of compact quantum groups, Comm. Math. Phys. 167 (1995), no. 3, 509-529.

Reiji Tomatsu, Department of Mathematical Sciences, University of Tokyo, 3-8-1 Komaba, Meguro, Tokyo, 153-8914, JAPAN

E-mail address: tomatsu@ms.u-tokyo.ac.jp 